\title{
RIGHT COIDEAL SUBALGEBRAS OF THE BOREL PART OF A QUANTIZED ENVELOPING ALGEBRA
}

\author{
ISTVÁN HECKENBERGER AND STEFAN KOLB
}

\begin{abstract}
For the Borel part of a quantized enveloping algebra we classify all right coideal subalgebras for which the intersection with the coradical is a Hopf algebra. The result is expressed in terms of characters of the subalgebras $U^{+}[w]$ of the quantized enveloping algebra, introduced by de Concini, Kac, and Procesi for any Weyl group element $w$. We explicitly determine all characters of $U^{+}[w]$ building on recent work by Yakimov on prime ideals of $U^{+}[w]$ which are invariant under a torus action.
\end{abstract}

\section{INTRODUCTION}

Let $\mathfrak{g}$ be a complex, finite-dimensional, semisimple Lie algebra and $U_{q}(\mathfrak{g})$ the corresponding quantized enveloping algebra. Quantum analogs of Lie subalgebras of $\mathfrak{g}$ are often realized as coideal subalgebras of $U_{q}(\mathfrak{g})$. Recall that a subalgebra $C$ of a Hopf algebra $H$ is called a right coideal subalgebra if the coproduct $\Delta$ of $H$ satisfies $\Delta(C) \subseteq C \otimes H$. The universal enveloping algebra $U(\mathfrak{g})$ is a cocommutative Hopf algebra and hence right coideal subalgebras of $U(\mathfrak{g})$ are always Hopf subalgebras. The quantum deformation $U_{q}(\mathfrak{g})$, however, is essentially obtained by deforming the coproduct. Hence one expects quantum deformations of Hopf subalgebras of $U(\mathfrak{g})$ inside $U_{q}(\mathfrak{g})$ only to satisfy the weaker coideal property.

Let $\Pi$ be a basis of simple roots for $\mathfrak{g}$. Let $U=U_{q}(\mathfrak{g})$ be defined as in Jan96, Chapter 4] over a base field $k$ with $q \in k \backslash\{0\}$ not a root of unity. Let $U^{+}$and $U^{0}$ denote the subalgebras of $U$ generated by the sets $\left\{E_{\alpha} \mid \alpha \in \Pi\right\}$ and $\left\{K_{\alpha}, K_{\alpha}^{-1} \mid \alpha \in\right.$ $\Pi\}$, respectively, and define the positive Borel part $U^{\geq 0}=U^{+} U^{0}$ which is a Hopf subalgebra of $U$. In the present paper we give an explicit combinatorial classification of all right coideal subalgebras $C$ of $U^{\geq 0}$ for which $C \cap U^{0}$ is a Hopf algebra. To be more precise, let $Q$ denote the root lattice and, for any element $w$ in the Weyl group $W$ of $\mathfrak{g}$, let $U^{+}[w]$ denote the subalgebra of $U^{+}$generated by the corresponding Lusztig root vectors as defined in [Jan96, 8.24]. Our first main result, which is made precise in Theorem 2.15, states the following:

There is a canonical bijection between the set of all right coideal subalgebras $C$ of $U^{\geq 0}$ for which $C \cap U^{0}$ is a Hopf algebra and the set of all triples $(w, \phi, L)$ where $w \in W, \phi: U^{+}[w] \rightarrow k$ is a character, and $L$ is a subgroup of $Q$ such that $\phi$ and $L$ satisfy an additional compatibility condition.

2000 Mathematics Subject Classification. 17B37.

Key words and phrases. Quantum groups, coideal subalgebras.

The work of I. Heckenberger was supported by DFG within a Heisenberg fellowship. The work of S. Kolb was supported by the School of Mathematics and the Maxwell Institute for Mathematical Sciences at the University of Edinburgh and by the Netherlands Organization for Scientific Research (NWO) within the VIDI-project "Symmetry and modularity in exactly solvable models". 
Many examples of coideal subalgebras of $U$ were initially constructed with a theory of homogeneous spaces or harmonic analysis for quantum groups in mind. Koornwinder's observation Koo93 that a quantum group analog of one-dimensional complex projective space can be obtained via a skew primitive element in $U_{q}\left(\mathfrak{s l}_{2}(\mathbb{C})\right.$ ) inspired the development of a theory of quantum symmetric spaces via coideal subalgebras. First, quantum analogs of all classical symmetric pairs were constructed in a case by case fashion Nou96, Dij96, NDS97. Later, a comprehensive theory of quantum symmetric pairs as one-sided coideal subalgebras in $U$ was developed by G. Letzter Let02, Let03. Letzter's work also contains general qualitative results about the structure of coideal subalgebras of $U$, mainly in terms of filtrations and the associated graded algebras [Let02, Section 4]. Closely related is a program initiated by Kharchenko to determine all right coideal subalgebras of $U^{\geq 0}$ which contain $U^{0}$. It was proved for $\mathfrak{g}$ of type $A_{n}$ [KS08, $B_{n}$ Kha09, and $G_{2}$ Pog09 that the number of such right coideal subalgebras coincides with the order of the Weyl group. Recently, the situation was clarified by Schneider and the first named author who proved in [HS09] that the algebras $U^{+}[w] U^{0}$, where $w \in W$, exhaust all right coideal subalgebras of $U^{\geq 0}$ which contain $U^{0}$. This result forms the starting point of the present work.

Motivated by the above classification, the second main result of this paper consists of an explicit combinatorial description of the set $\operatorname{Char}\left(U^{+}[w]\right)$ of all characters of $U^{+}[w]$. Define $W \leq w=\{y \in W \mid y \leq w\}$ where $\leq$ denotes the Bruhat order on $W$. In Sections 3.3 and 3.5 we show the following:

The set $\operatorname{Char}\left(U^{+}[w]\right)$ can be canonically identified with a disjoint union of spectra of Laurent polynomial rings indexed by the elements of a subset $W^{w} \subseteq W \leq w$. The subset $W^{w}$ is given explicitly, and the dimension of the component corresponding to $y \in W^{w}$ is $\ell(w)-\ell(y)$, where $\ell$ denotes the length function.

To determine Char $\left(U^{+}[w]\right)$ we apply the theory of $H$-stable prime ideals, outlined in [BG02, Part II]. Here $H=(k \backslash\{0\})^{\operatorname{rank}(\mathfrak{g})}$ is a torus which naturally acts on $U^{+}[w]$. A subspace of $U^{+}[w]$ is $H$-stable if and only if it is naturally graded by the root lattice $Q$. Following a standard construction, one associates an $H$-prime ideal of $U^{+}[w]$ to any character of $U^{+}[w]$. Recently, Yakimov obtained an order preserving bijection between $W^{\leq w}$ with the Bruhat order and the set of $H$-prime ideals of $U^{+}[w]$, which is ordered by inclusion [Yak09]. To determine $\operatorname{Char}\left(U^{+}[w]\right)$ we directly find a large family of $H$-prime ideals which correspond to characters. We then apply Yakimov's result and previous results by Gorelik Gor00 to show that this family is complete.

Characters of $U^{+}[w]$ appear in the classification of right coideal subalgebras via a standard construction: For any right coideal subalgebra $C$ of a Hopf algebra $H$ and any character $\phi: C \rightarrow k$ one obtains a new right coideal subalgebra $C_{\phi}$ by application of the coproduct and evaluation of $\phi$ on the first tensor component (cf. Section 2.3). It was recently noted that this construction is also at the heart of coideal subalgebras of $U$ which appear in the theory of quantum symmetric pairs KS09, Section 4]. One might ask if $U$ possesses certain standard right coideal subalgebras from which all other right coideal subalgebras are obtained via characters in this way. This is but one question on the way towards a general combinatorial classification of right coideal subalgebras of $U$.

The classification of right coideal subalgebras of $U^{\geq 0}$ which contain $U^{0}$ also holds for small quantum groups of semisimple Lie algebras where $q$ is a root of 
unity KS08, [HS09. In the setting of the present paper, however, the assumption that $q$ is not a root of unity is essential. It would be interesting to extend the classification of right coideal subalgebras to more general classes of pointed Hopf algebras.

This paper consists of the two sections outlined above and an appendix. The appendix contains technical results on root systems and Weyl group combinatorics which are used to prove the statements leading up to Theorem 3.17.

\section{Right Coideal Subalgebras of $U \geq 0$}

2.1. Quantized enveloping algebras and right coideal subalgebras. We mostly follow the notation and conventions of [Jan96. Let $\mathfrak{g}$ be a finite-dimensional complex semisimple Lie algebra and let $\Phi$ be the root system with respect to a fixed Cartan subalgebra. We also fix a basis $\Pi$ of $\Phi$ and denote by $\Phi^{+}$and $\Phi^{-}$the corresponding sets of positive roots and negative roots, respectively. Let $W$ be the Weyl group of $\mathfrak{g}$ and let $(\cdot, \cdot)$ be the invariant scalar product on the real vector space generated by $\Pi$ such that $(\alpha, \alpha)=2$ for all short roots in each component. For any $\beta \in \Phi$ we write $s_{\beta}$ to denote the reflection at the hyperplane orthogonal to $\beta$ with respect to $(\cdot, \cdot)$. Let $Q=\mathbb{Z} \Pi$ be the root lattice and let $Q_{+}=\mathbb{N}_{0} \Pi$. For each $\alpha \in \Pi$ let $d_{\alpha}=(\alpha, \alpha) / 2$. Let $U=U_{q}(\mathfrak{g})$ be the quantized enveloping algebra of $\mathfrak{g}$ in the sense of [Jan96, Chapter 4]. More precisely, let $k$ be a field and fix an element $q \in k$ with $q \neq 0$ and $q^{n} \neq 1$ for all $n \in \mathbb{N}$. Then $U$ is the unital associative algebra defined over $k$ with generators $K_{\alpha}, K_{\alpha}^{-1}, E_{\alpha}, F_{\alpha}$ for all $\alpha \in \Pi$ and relations given in [Jan96, 4.3]. By [Jan96, Proposition 4.11] there is a unique Hopf algebra structure on $U$ with coproduct $\Delta$, counit $\varepsilon$, and antipode $S$ such that

$$
\begin{aligned}
& \Delta\left(E_{\alpha}\right)=E_{\alpha} \otimes 1+K_{\alpha} \otimes E_{\alpha}, \quad \varepsilon\left(E_{\alpha}\right)=0, \quad S\left(E_{\alpha}\right)=-K_{\alpha}^{-1} E_{\alpha}, \\
& \Delta\left(F_{\alpha}\right)=F_{\alpha} \otimes K_{\alpha}^{-1}+1 \otimes F_{\alpha}, \quad \varepsilon\left(F_{\alpha}\right)=0, \quad S\left(F_{\alpha}\right)=-F_{\alpha} K_{\alpha}, \\
& \Delta\left(K_{\alpha}\right)=K_{\alpha} \otimes K_{\alpha}, \quad \varepsilon\left(K_{\alpha}\right)=1, \quad S\left(K_{\alpha}\right)=K_{\alpha}^{-1} .
\end{aligned}
$$

We will make use of Sweedler notation for the coproduct in the form $\Delta(x)=$ $x_{(1)} \otimes x_{(2)}$ for any $x \in U$. Let ad denote the left adjoint action of $U$ on itself, that is, $(\operatorname{ad} x)(y)=x_{(1)} y S\left(x_{(2)}\right)$ for all $x, y \in U$.

As in [Jan96, Chapter 4] let $U^{+}, U^{0}$, and $U^{\geq 0}$ be the subalgebras of $U$ generated by the sets $\left\{E_{\alpha} \mid \alpha \in \Pi\right\},\left\{K_{\alpha}, K_{\alpha}^{-1} \mid \alpha \in \Pi\right\}$ and $\left\{K_{\alpha}, K_{\alpha}^{-1}, E_{\alpha} \mid \alpha \in \Pi\right\}$, respectively. In fact, $U^{0}$ and $U \geq 0$ are Hopf subalgebras of $U_{q}(\mathfrak{g})$. For any $\beta \in Q$ define $K_{\beta}=\prod_{\alpha \in \Pi} K_{\alpha}^{n_{\alpha}}$ if $\beta=\sum_{\alpha \in \Pi} n_{\alpha} \alpha$ for some $n_{\alpha} \in \mathbb{Z}$.

For any $\alpha \in \Pi$ let $T_{\alpha}$ denote the algebra automorphisms of $U$ defined in Jan96, 8.14]. Let $w \in W$ be an element of length $\ell(w)=t$ and choose $\alpha_{1}, \ldots, \alpha_{t} \in \Pi$ such that $s_{\alpha_{1}} s_{\alpha_{2}} \cdots s_{\alpha_{t}}$ is a reduced expression of $w$. For all $i \in\{1,2, \ldots, t\}$ let $\beta_{i}=s_{\alpha_{1}} \cdots s_{\alpha_{i-1}} \alpha_{i}$. In [Jan96, 8.21] Lusztig root vectors in $U^{+}$are defined by $E_{\beta_{i}}=T_{\alpha_{1}} \cdots T_{\alpha_{i-1}} E_{\alpha_{i}}$ for all $i \in\{1, \ldots, t\}$. Following [CKP95] the subspace

$$
U^{+}[w]=\operatorname{span}_{k}\left\{E_{\beta_{t}}^{a_{t}} \cdots E_{\beta_{2}}^{a_{2}} E_{\beta_{1}}^{a_{1}} \mid a_{1}, \ldots, a_{t} \in \mathbb{N}_{0}\right\},
$$

is attached to $w$ in [Jan96, 8.24]. It is shown in CKP95] that $U^{+}[w]$ is a subalgebra which does not depend on the reduced expression. The following observation is the starting point of our investigations of right coideal subalgebras of $U \geq 0$.

Theorem 2.1. [HS09, Theorem 7.3] The map from $W$ to the set of right coideal subalgebras of $U^{\geq 0}$ containing $U^{0}$, given by $w \mapsto U^{+}[w] U^{0}$, is a bijection. 
For all $\alpha \in Q_{+}$let

$$
U_{\alpha}^{+}=\left\{x \in U^{+} \mid K_{\beta} x K_{\beta}^{-1}=q^{(\beta, \alpha)} x \quad \text { for all } \beta \in Q\right\} .
$$

The algebra $U^{\geq 0}$ admits a $Q^{2}$-grading given by

$$
U^{\geq 0}=\underset{\alpha, \beta \in Q}{\oplus} U_{\alpha}^{+} K_{\beta} .
$$

For all $\alpha, \beta \in Q$ let $\operatorname{pr}_{(\alpha, \beta)}: U^{\geq 0} \rightarrow U_{\alpha}^{+} K_{\beta}$ be the unique $Q^{2}$-graded projection. Note that $\operatorname{pr}_{(\alpha, \beta)}=0$ if $\alpha \in Q \backslash Q_{+}$.

A subspace $T$ of $U^{0}$ is a Hopf subalgebra if and only if there exists a subgroup $L$ of the abelian group $Q$ such that $T=\operatorname{span}_{k}\left\{K_{\alpha} \mid \alpha \in L\right\}$. We write $T_{L}$ for this Hopf algebra. In particular, $T_{\{0\}}=k$ and $T_{Q}=U^{0}$. We will frequently use the notation

$$
L^{\perp}=\{\gamma \in Q \mid(\gamma, \beta)=0 \text { for all } \beta \in L\}
$$

to denote the orthogonal complement of a subgroup $L \subseteq Q$. The adjoint action of $T_{L}$ on $U^{\geq 0}$ is diagonalizable. We say that an element $x \in U^{\geq 0} \backslash\{0\}$ is a weight vector for ad $T_{L}$, if there exists $\alpha \in Q$ such that

$$
\left(\operatorname{ad} K_{\beta}\right)(x)=q^{(\beta, \alpha)} x \quad \text { for all } \beta \in L .
$$

Any ad $T_{L}$-stable subspace of $U^{\geq 0}$ has a basis consisting of weight vectors for ad $T_{L}$.

Lemma 2.2. Let $L \subseteq Q$ be a subgroup and $\alpha_{1}, \alpha_{2}, \beta_{1}, \beta_{2} \in Q$. Let $x \in U \geq 0$ be a weight vector for ad $T_{L}$ such that $\operatorname{pr}_{\left(\alpha_{1}, \beta_{1}\right)}(x) \neq 0$ and $\operatorname{pr}_{\left(\alpha_{2}, \beta_{2}\right)}(x) \neq 0$. Then $\alpha_{1}-\alpha_{2} \in L^{\perp}$.

Proof. The assumptions of the Lemma together with the direct sum decomposition (2.5) imply that $\left(\operatorname{ad} K_{\beta}\right)(x)=q^{\left(\alpha_{1}, \beta\right)} x=q^{\left(\alpha_{2}, \beta\right)} x$ for all $\beta \in L$. As $q$ is not a root of unity one obtains $\left(\alpha_{1}-\alpha_{2}, \beta\right)=0$ for all $\beta \in L$.

For any right coideal $C \subseteq U^{\geq 0}$ and any $\beta \in Q$ define

$$
C_{\beta}=U^{+} K_{\beta} \cap C .
$$

The following lemma is an adapted version of Let02, Lemmata 1.1, 1.3].

Lemma 2.3. Let $C \subseteq U^{\geq 0}$ be a right coideal. Then $C=\oplus_{\beta \in Q} C_{\beta}$. If $C$ is an algebra then this decomposition is an algebra grading of $C$ by $Q$.

Proof. Let $p: U^{\geq 0} \rightarrow U^{0}, p=\oplus_{\beta \in Q} \operatorname{pr}_{(0, \beta)}$, be the unique $Q^{2}$-graded projection. As $p$ is an algebra map, Equations (2.1) and (2.3) imply that $p$ is a coalgebra homomorphism. Since $C$ is a right coideal, the map $p^{\prime}=($ id $\otimes p) \Delta: U^{\geq 0} \rightarrow$ $U^{\geq 0} \otimes U^{0}$ induces a right coaction of $U^{0}$ on $C$. Further,

$$
p^{\prime}\left(x K_{\beta}\right)=x K_{\beta} \otimes K_{\beta} \quad \text { for all } \beta \in Q, \quad x \in U^{+},
$$

and hence $C=\oplus_{\beta \in Q} C_{\beta}$. The last claim of the lemma follows now from the commutation relations in $U^{\geq 0}$.

The comultiplication $\Delta$ of $U^{\geq 0}$ is compatible with the $Q^{2}$-grading (2.5) via

$$
\Delta(x)-x \otimes K_{\beta} \in \underset{\gamma \prec \alpha}{\oplus} U_{\gamma}^{+} K_{\alpha+\beta-\gamma} \otimes U_{\alpha-\gamma}^{+} K_{\beta}
$$

for $x \in U_{\alpha}^{+} K_{\beta}, \alpha, \beta \in Q$, where $\gamma \prec \alpha$ means that $\alpha-\gamma \in Q_{+} \backslash\{0\}$. The relation $\prec$ defines a partial ordering on $Q$.

The next lemmata show that left coideals are useful for the study of right coideals. 
Lemma 2.4. Let $C \subseteq U \geq 0$ be a right coideal. Let $\beta \in Q$ and $x \in C_{\beta} \backslash\{0\}$. Let $J$ be a $Q^{2}$-homogeneous subspace and a left coideal of $U \geq 0$. Let $\gamma \in Q$ be maximal with respect to $\prec$ such that $\operatorname{pr}_{(\gamma, \beta)}(x) \notin J$. Then $K_{\beta+\gamma} \in C$.

Proof. For all $\alpha \in Q_{+}$let $x_{\alpha} \in U_{\alpha}^{+}$be such that $x=\sum_{\alpha \in Q_{+}} x_{\alpha} K_{\beta}$. Let $p_{J}: U^{\geq 0} \rightarrow$ $U \geq 0 / J$ be the canonical map of left $U^{\geq 0}$-comodules. Since $J$ is $Q^{2}$-homogeneous, the map $p_{J}$ is $Q^{2}$-graded. Let $\alpha \in Q_{+}$with $\gamma \prec \alpha$. By assumption, $x_{\alpha} K_{\beta} \in J$. Since $J$ is a left coideal, we conclude that (id $\left.\otimes p_{J}\right) \Delta\left(x_{\alpha} K_{\beta}\right)=0$. Thus the maximality of $\gamma$ and Relation (2.8) imply that

$$
\begin{aligned}
\left(\mathrm{id} \otimes p_{J}\right) \Delta(x)= & K_{\beta+\gamma} \otimes p_{J}\left(x_{\gamma} K_{\beta}\right) \\
& + \text { terms } x_{1} \otimes p_{J}\left(x_{2}\right) \text { with } x_{2} \in U_{\alpha}^{+} K_{\beta} \text { for some } \alpha \neq \gamma .
\end{aligned}
$$

Since $C$ is a right coideal one obtains $K_{\beta+\gamma} \in C$.

Lemma 2.5. Let $C \subseteq U^{\geq 0}$ be a right coideal and let $x \in C_{-\beta}$ for some $\beta \in Q$. Let $L \subseteq Q$ be a subgroup such that $C \cap U^{0} \subseteq T_{L}$. Assume that $x$ is a weight vector for $\operatorname{ad} T_{L}$. Let $\alpha \in Q_{+}$be maximal with respect to $\prec$ such that $x_{\alpha}:=\operatorname{pr}_{(\alpha,-\beta)}(x) \neq 0$. Let $J_{\alpha} \subseteq U \geq 0$ be the left coideal generated by $x_{\alpha}$.

(1) If $\gamma \in Q$ satisfies $x_{\gamma}:=\operatorname{pr}_{(\gamma,-\beta)}(x) \neq 0$ then $x_{\gamma} \in J_{\alpha}$ and $\alpha-\gamma \in L^{\perp} \cap Q_{+}$.

(2) The element $\alpha \in Q_{+}$is uniquely determined by $x$.

(3) If $\operatorname{pr}_{(\gamma,-\beta)}(x) \neq 0$ for some $\gamma \in \beta+L^{\perp}$ then $\beta=\alpha$.

Proof. Since $x_{\alpha}$ is $Q^{2}$-homogeneous the subspace $J_{\alpha}$ of $U^{\geq 0}$ is also $Q^{2}$-homogeneous by Relation (2.8). Let $\gamma \in Q$ be such that $x_{\gamma}:=\operatorname{pr}_{(\gamma,-\beta)}(x) \neq 0$. Then $\alpha-\gamma \in L^{\perp}$ by Lemma 2.2. We now proceed indirectly to prove the first statement. If $x_{\gamma} \notin J_{\alpha}$ and $\gamma$ is maximal with respect to $\prec$ with this property, then Lemma 2.4 for $J=J_{\alpha}$ implies $K_{\gamma-\beta} \in C$. Similarly, $K_{\alpha-\beta} \in C$ by Lemma 2.4 for $J=0$. Hence $\gamma-\alpha \in L$ and thus $\alpha=\gamma$ as $(\cdot, \cdot)$ is positive definite and $\alpha-\gamma \in L^{\perp}$. This however is a contradiction to $x_{\alpha} \in J_{\alpha}, x_{\gamma} \notin J_{\alpha}$. Clearly, $x_{\gamma} \in J_{\alpha}$ implies that $\alpha-\gamma \in Q_{+}$.

Statement (2) immediately follows from (1). To prove statement (3) assume that $\operatorname{pr}_{(\gamma,-\beta)}(x) \neq 0$ for some $\gamma \in \beta+L^{\perp}$. By (1) one gets $\alpha-\beta \in L^{\perp}$. On the other hand Lemma 2.4 implies that $\alpha-\beta \in L$ and hence $\alpha=\beta$.

2.2. Connected right coideal subalgebras. In analogy to the terminology for coalgebras [Mon93, 5.1.5] we make the following definition.

Definition 2.6. Let $C$ be a right coideal subalgebra of $U^{\geq 0}$. We say that $C$ is connected if $C \cap U^{0}=k 1$.

Let $\mathfrak{K}$ denote the set of right coideal subalgebras $C$ of $U^{\geq 0}$ such that $C \cap U^{0}$ is a Hopf algebra. For any $C \in \mathfrak{K}$ we write $L(C)$ for the subgroup of $Q$ corresponding to the Hopf subalgebra $C \cap U^{0}$ of $U^{0}$. Clearly, $C$ is connected if and only if $L(C)=0$.

We will now show that any right coideal subalgebra $C \in \mathfrak{K}$ decomposes into the product of $T_{L(C)}$ and a connected right coideal subalgebra. To this end define for any $C \in \mathfrak{K}$ a subspace

$$
I(C)=\underset{\beta \in Q_{+}}{\oplus}\left\{x \in C_{-\beta} \mid\left(\operatorname{ad} K_{\alpha}\right)(x)=q^{(\alpha, \beta)} x \text { for all } \alpha \in L(C)\right\} .
$$

The definition implies directly that

$$
I(C)=\underset{\beta \in Q_{+}}{\oplus}\left\{x \in C_{-\beta} \mid \operatorname{pr}_{(\gamma,-\beta)}(x)=0 \quad \text { for all } \gamma \in Q \backslash\left(\beta+L(C)^{\perp}\right)\right\} .
$$


Lemma 2.7. Let $C \in \mathfrak{K}, \beta \in Q_{+}$, and let $x \in I(C) \cap C_{-\beta}$ with $x \neq 0$. Then $\operatorname{pr}_{(\beta,-\beta)}(x) \neq 0$. If $\operatorname{pr}_{(\gamma,-\beta)}(x) \neq 0$ for some $\gamma \in Q$ then $\beta-\gamma \in L(C)^{\perp} \cap Q_{+}$.

Proof. Let $\alpha$ be the unique maximal element in $Q$ with $\operatorname{pr}_{(\alpha,-\beta)}(x) \neq 0$, see Lemma 2.5(2). Then $\alpha \in \beta+L(C)^{\perp}$ by Equation (2.10), and hence $\alpha=\beta$ by Lemma 2.5 (3). The remaining claim holds by Lemma 2.5(1).

Proposition 2.8. Let $C \in \mathfrak{K}$.

(1) $I(C)$ is a connected, ad $T_{L(C)}$-stable right coideal subalgebra of $U^{\geq 0}$.

(2) The decomposition $I(C)=\oplus_{\beta \in Q_{+}} I(C)_{-\beta}$ is an algebra grading.

(3) The multiplication map $I(C) \otimes U^{0} \rightarrow U^{\geq 0}$ is injective.

(4) The multiplication map $I(C) \otimes T_{L(C)} \rightarrow C$ is bijective.

(5) Let $D$ be a connected ad $T_{L(C)}$-stable right coideal subalgebra of $U^{\geq 0}$. If $D \subseteq C$ then $D \subseteq I(C)$. If $D T_{L(C)}=C$ then $D=I(C)$.

Proof. (1) The $Q^{2}$-grading of $U^{\geq 0}$ is an algebra grading, and hence $I(C)$ is a subalgebra of $C$. Relation (2.8) and Equation (2.10) imply that $\Delta(I(C)) \subseteq I(C) \otimes U \geq 0$. Let $\beta \in Q$ and let $x \in I(C) \cap C_{-\beta} \cap U^{0}$. If $x \neq 0$ then $\operatorname{pr}_{(\beta,-\beta)}(x) \neq 0$ by Lemma 2.7 and hence $\beta=0$. Thus $I(C) \cap U^{0}=k 1$, and hence $I(C)$ is a connected right coideal subalgebra of $C$. Further, $I(C)$ is ad $T_{L(C)}$-stable by Definition (2.9).

(2) This is a special case of Lemma 2.3.

(3) Consider elements $x_{\beta} \in I(C)_{-\beta}$ and $t_{\beta} \in U^{0} \backslash\{0\}$ such that $x_{\beta} \neq 0$ for finitely many $\beta \in Q_{+}$. Choose a maximal $\gamma \in Q_{+}$such that $x_{\gamma} \neq 0$. Then $\operatorname{pr}_{(\gamma,-\gamma)}\left(x_{\gamma}\right) \neq 0$ but $\operatorname{pr}_{(\gamma, \delta)}\left(x_{\beta}\right)=0$ for all $\beta \in Q_{+} \backslash\{\gamma\}, \delta \in Q$ by Lemma 2.7 Hence $\operatorname{pr}_{(\gamma, \delta)}\left(\sum_{\beta} x_{\beta} t_{\beta}\right)=\operatorname{pr}_{(\gamma, \delta)}\left(x_{\gamma} t_{\gamma}\right) \neq 0$ for a suitable $\delta \in Q$. This implies $\sum_{\beta} x_{\beta} t_{\beta} \neq 0$ which proves injectivity.

(4) Injectivity follows from (3). To verify surjectivity consider an element $x \in$ $C_{-\beta}$ for some $\beta \in Q$ and assume that $x$ is a weight vector for ad $T_{L(C)}$. As in Lemma 2.5 let $\alpha \in Q_{+}$be maximal such that $\operatorname{pr}_{(\alpha,-\beta)}(x) \neq 0$. Lemma 2.4 implies that $K_{\alpha-\beta} \in T_{L(C)}$. Moreover, $x=x K_{\beta-\alpha} K_{\alpha-\beta}$ and $x K_{\beta-\alpha} \in C_{-\alpha} \cap I(C)$ by Equation (2.9). Hence $x$ lies indeed in the image of the multiplication map.

(5) Assume that $D \subseteq C$. By (2.7) one has $D_{-\beta} \subseteq C_{-\beta}$ for all $\beta \in Q$. Further, $D_{-\beta}$ is ad $T_{L(C)}$-stable for all $\beta \in Q$ since $D$ and $U^{+} K_{-\beta}$ are ad $T_{L(C)}$-stable. Let $x \in D_{-\beta}$ be a weight vector for $\operatorname{ad} T_{L(C)}$. By Lemma 2.5 there exists a unique maximal weight $\alpha \in Q$ such that $\operatorname{pr}_{(\alpha,-\beta)} \neq 0$. Since $D$ is connected Lemma 2.4 implies $\alpha=\beta$. Thus $x \in I(C)$ by Equation (2.9). Since ad $T_{L(C)}$ is diagonalizable, we conclude that $D \subseteq I(C)$. Then the last claim holds by (4).

Corollary 2.9. Let $C \in \mathfrak{K}$ and let $L \subseteq Q$ be a subgroup. Assume that $C$ is $\operatorname{ad} T_{L}$-stable. Then $C T_{L} \in \mathfrak{K}$.

Proof. It follows from the assumptions that $C T_{L}$ is a right coideal subalgebra of $U \geq 0$. Further, $C T_{L}=I(C) T_{L(C)+L}$ by Proposition 2.8(4), and hence $C T_{L} \cap U^{0}=$ $T_{L(C)+L}$ by Proposition 2.8 (3). Thus $C T_{L} \in \mathfrak{K}$.

Define a linear map

$$
\varphi: U^{\geq 0} \rightarrow U^{\geq 0}, \quad \varphi(x)=\sum_{\beta \in Q} \operatorname{pr}_{(\beta,-\beta)}(x)
$$


and observe that $\varphi$ is a homomorphism of right $U^{\geq 0}$-comodules by Relation (2.8). For all $C \in \mathfrak{K}$ define

$$
\bar{C}=\varphi(I(C)) U^{0}
$$

We will see in the following proposition that $\bar{C}$ is a right coideal subalgebra of $U^{\geq 0}$ containing $U^{0}$ as in Theorem 2.1. For any $C \in \mathfrak{K}$ let $\varphi_{C}: I(C) \rightarrow U \geq 0$ be the restriction of $\varphi$ to $I(C)$.

Proposition 2.10. Let $C \in \mathfrak{K}$. Then the following hold.

(1) $\varphi_{C}$ is an injective homomorphism of right $U^{\geq 0}$-comodule algebras.

(2) $\varphi_{C}(I(C))$ is a connected, ad $U^{0}$-stable right coideal subalgebra of $U \geq 0$.

(3) $\bar{C} \in \mathfrak{K}$, and the multiplication map $\varphi_{C}(I(C)) \otimes U^{0} \rightarrow \bar{C}$ is bijective.

(4) $\varphi_{C}(I(C))=S\left(U^{+}\right) \cap \bar{C}$.

Proof. (1) Lemma 2.7 implies that $\varphi_{C}$ is injective and that

$$
\operatorname{pr}_{(\beta,-\beta)}(x) \operatorname{pr}_{(\gamma,-\gamma)}(y)=\operatorname{pr}_{(\beta+\gamma,-(\beta+\gamma))}(x y) \quad \text { for all } x \in I(C)_{-\beta}, y \in I(C)_{-\gamma} .
$$

This and Proposition 2.8 (2) imply that $\varphi_{C}$ is an algebra homomorphism. As $\varphi$ is a homomorphism of right $U^{\geq 0}$-comodules so is $\varphi_{C}$.

(2) By (1) the subspace $\varphi_{C}(I(C))$ is a right coideal subalgebra of $U \geq 0$. It is connected since $\varphi\left(U^{\geq 0}\right) \cap U^{0}=k 1$. It is ad $U^{0}$-stable, since $\varphi_{C}\left(I(C)_{-\beta}\right)$ is ad $U^{0}$ stable for all $\beta \in Q_{+}$.

Recall that the multiplication map $S\left(U^{+}\right) \otimes U^{0} \rightarrow U^{\geq 0}$ is bijective. Thus (3) and (4) follow from $(2)$ and since $\varphi_{C}(I(C)) \subseteq S\left(U^{+}\right)$.

Define a linear map $\psi: U^{+} \rightarrow S\left(U^{+}\right)$by

$$
\psi\left(x_{\beta}\right)=q^{-(\beta, \beta) / 2} x_{\beta} K_{\beta}^{-1} \quad \text { for all } x_{\beta} \in U_{\beta}^{+}, \beta \in Q_{+} .
$$

The following technical Lemma will allow us to identify the connected ad $U^{0}$-stable right coideal subalgebras of $U^{\geq 0}$ in Theorem 2.12

Lemma 2.11. The map $\psi: U^{+} \rightarrow S\left(U^{+}\right)$is an algebra isomorphism. Moreover, $\psi\left(U^{+}[w]\right)=S\left(U^{+}\right) \cap U^{+}[w] U^{0}$ for all $w \in W$.

Proof. Since $S\left(U^{+}\right)$is generated by the elements $S\left(E_{\alpha}\right)=-K_{\alpha}^{-1} E_{\alpha}$, where $\alpha \in \Pi$, we conclude that $S\left(U^{+}\right)$is spanned by the elements of the form $x_{\beta} K_{\beta}^{-1}$, where $\beta \in Q_{+}$and $x_{\beta} \in U_{\beta}^{+}$. Thus $\psi\left(U^{+}\right)=S\left(U^{+}\right)$. Since $U^{+}$is graded by $Q_{+}$, the map $\psi$ is also injective. For any $\alpha, \beta \in Q_{+}$and any $x_{\alpha} \in U_{\alpha}^{+}, y_{\beta} \in U_{\beta}^{+}$one verifies that $\psi\left(x_{\alpha} y_{\beta}\right)=\psi\left(x_{\alpha}\right) \psi\left(y_{\beta}\right)$ which implies that $\psi$ is an algebra homomorphism.

Let $w \in W$. Since $U^{+}[w]$ is graded by $Q_{+}$, the last claim follows from the definition of $\psi$ and the above description of $S\left(U^{+}\right)$.

Theorem 2.12. (1) Let $w \in W$. Then $\psi\left(U^{+}[w]\right)$ is a connected ad $U^{0}$-stable right coideal subalgebra of $U^{\geq 0}$.

(2) Any connected ad $U^{0}$-stable right coideal subalgebra of $U \geq 0$ is of the form $\psi\left(U^{+}[w]\right)$ for a unique $w \in W$.

Proof. (1) Let $C=U^{+}[w] U^{0}$. Then $C \in \mathfrak{K}$ by Theorem 2.1. Moreover, $L(C)=$ $Q$ and hence $I(C)=S\left(U^{+}\right) \cap U^{+}[w] U^{0}$ by Equation (2.9) or Equation (2.10). Therefore $I(C)=\psi\left(U^{+}[w]\right)$ by Lemma 2.11. Moreover, $\varphi_{C}=\mathrm{id}_{I(C)}$, and hence the claim holds by Proposition $2.10(2)$. 
(2) Let now $D$ be a connected ad $U^{0}$-stable right coideal subalgebra. Then $I(D)=D$ by Proposition 2.8(4), and hence $\varphi_{D}=\mathrm{id}_{D}$ by Equation (2.9). Moreover $\bar{D}=D U^{0} \in \mathfrak{K}$ by Proposition 2.10 (3), and $D=S\left(U^{+}\right) \cap D U^{0}$ by Proposition 2.10(4). By Theorem 2.1, $D U^{0}=U^{+}[w] U^{0}$ for a unique $w \in W$. Thus $D=$ $\psi\left(U^{+}[w]\right)$ by Lemma 2.11

2.3. Right coideal subalgebras and characters. By definition a character of an associative, unital $k$-algebra $A$ is an algebra homomorphism $\phi: A \rightarrow k$ with $\phi(1)=$ 1. Let $\operatorname{Char}(A)$ denote the set of characters of $A$. The following construction, though only considered for $U^{\geq 0}$ here, works for right coideal subalgebras of an arbitrary Hopf algebra.

Let $C$ be a right coideal subalgebra of $U^{\geq 0}$ and $\phi \in \operatorname{Char}(C)$. Consider the subspace

$$
C_{\phi}:=\{(\phi \otimes \mathrm{id}) \Delta(x) \mid x \in C\}=\left\{\phi\left(x_{(1)}\right) x_{(2)} \mid x \in C\right\}
$$

of $U \geq 0$. It follows from the coassociativity of the coproduct that $C_{\phi}$ is a right coideal subalgebra of $U \geq 0$ and that the map

$$
C \rightarrow C_{\phi}, \quad x \mapsto \phi\left(x_{(1)}\right) x_{(2)} \quad \text { for all } x \in U^{\geq 0}
$$

is a surjective homomorphism of right $U^{\geq 0}$-comodule algebras.

Lemma 2.13. Let $C$ be a connected right coideal subalgebra of $U^{\geq 0}$ and $\phi \in$ Char $(C)$. Then $C_{\phi}$ is connected and the map (2.11) is an isomorphism.

Proof. Recall the standard $\mathbb{N}_{0}$-filtration of $U^{\geq 0}$. More explicitly, define a $\mathbb{Z}$-linear homomorphism ht : $Q \rightarrow \mathbb{Z}$ by $\operatorname{ht}(\alpha)=1$ for all $\alpha \in \Pi$. Now set $\mathcal{F}^{n} U^{\geq 0}=$ $\oplus_{\beta \in Q, \text { ht }(\beta) \leq n} U_{\beta}^{+} U^{0}$. Note that $\mathcal{F}$ is a filtration of $U^{\geq 0}$ both as an algebra and as a coalgebra. Assume that $C$ is connected. It suffices to show for any $x \in C$ and any $m \in \mathbb{N}_{0}$ the relation

$$
x \in \mathcal{F}^{m} U^{\geq 0} \backslash \mathcal{F}^{m-1} U^{\geq 0} \Longrightarrow \phi\left(x_{(1)}\right) x_{(2)} \notin \mathcal{F}^{m-1} U^{\geq 0} .
$$

To this end write $x=x_{m}+u$ with $x_{m} \in \oplus_{\beta \in Q \text {, ht }(\beta)=m} U_{\beta}^{+} U^{0}$ and $u \in \mathcal{F}^{m-1} U^{\geq 0}$. Since $\mathcal{F}$ is a filtration of coalgebras and $C$ is a connected right coideal subalgebra one obtains $\Delta\left(x_{m}\right)-1 \otimes x_{m} \in U^{\geq 0} \otimes \mathcal{F}^{m-1} U^{\geq 0}$. Thus $\phi\left(x_{(1)}\right) x_{(2)}-x_{m} \in \mathcal{F}^{m-1} U^{\geq 0}$ which implies $\phi\left(x_{(1)}\right) x_{(2)} \notin \mathcal{F}^{m-1} U^{\geq 0}$ and concludes the proof.

Remark 2.14. One can show that the map (2.11) is bijective for all $C \in \mathfrak{K}$.

Let $V \subseteq U^{+}$be an ad $U^{0}$-stable subspace and let $\phi \in \operatorname{Hom}(V, k)$ be a linear functional. We define

$$
\operatorname{supp} \phi=\left\{\beta \in Q_{+} \mid \phi(x) \neq 0 \text { for some } x \in U_{\beta}^{+} \cap V\right\} .
$$

We will only use the notion $\operatorname{supp} \phi$ in the case where $V=U^{+}[w]$ for some $w \in W$ and where $\phi$ is a character. The following theorem is the first main result of this paper. Recall that $\mathfrak{K}$ denotes the set of all right coideal subalgebras $C$ of $U^{\geq 0}$ such that $C \cap U^{0}$ is a Hopf algebra. Recall moreover that $\varepsilon$ denotes the counit of $U^{\geq 0}$.

Theorem 2.15. (1) Let $C \in \mathfrak{K}$. Then $\varphi_{C}(I(C))=\psi\left(U^{+}\left[w_{C}\right]\right)$ for a unique $w_{C} \in$ $W$, and $\varphi_{C}^{-1} \psi: U^{+}\left[w_{C}\right] \rightarrow I(C)$ is an algebra isomorphism with $\varphi_{C}^{-1} \psi\left(U^{+}\left[w_{C}\right] \cap\right.$ $\left.U_{\beta}^{+}\right)=I(C)_{-\beta}$ for all $\beta \in Q_{+}$. The map

$$
\phi_{C}: U^{+}\left[w_{C}\right] \rightarrow k, \quad \phi_{C}(x)=\varepsilon\left(\varphi_{C}^{-1} \psi(x)\right) \quad \text { for all } x \in U^{+}\left[w_{C}\right],
$$


is a character, and $L(C) \subseteq\left(\operatorname{supp} \phi_{C}\right)^{\perp}$.

(2) Let $w \in W, \phi \in \operatorname{Char}\left(U^{+}[w]\right)$, and $L \subseteq(\operatorname{supp} \phi)^{\perp}$ a subgroup. Let

$$
D(w, \phi):=\psi\left(U^{+}[w]\right)_{\phi \psi^{-1}}=\left\{\phi\left(\psi^{-1}\left(x_{(1)}\right)\right) x_{(2)} \mid x \in \psi\left(U^{+}[w]\right)\right\}
$$

and $C(w, \phi, L):=D(w, \phi) T_{L}$. Then $D(w, \phi)$ is a connected, ad $T_{L}$-stable right coideal subalgebra of $U^{\geq 0}$, and $C(w, \phi, L) \in \mathfrak{K}$.

(3) The map

$$
\begin{aligned}
\mathfrak{K} & \rightarrow\left\{(w, \phi, L) \mid w \in W, \phi \in \operatorname{Char}\left(U^{+}[w]\right), L \text { is a subgroup of }(\operatorname{supp} \phi)^{\perp}\right\} \\
C & \mapsto\left(w_{C}, \phi_{C}, L(C)\right)
\end{aligned}
$$

given by (1) is a bijection with inverse map $(w, \phi, L) \mapsto C(w, \phi, L)$ as in (2).

Proof. (1) By Proposition 2.10 (2), $\varphi_{C}(I(C))$ is a connected ad $U^{0}$-stable right coideal subalgebra of $U^{\geq 0}$, and hence $\varphi_{C}(I(C))=\psi\left(U^{+}\left[w_{C}\right]\right)$ for a unique $w_{C} \in W$ by Theorem 2.12 Now $\varphi_{C}$ is an injective algebra homomorphism by Proposition 2.10(1), and hence $\varphi_{C}^{-1} \psi$ is an algebra isomorphism. The compatibility of $\varphi_{C}^{-1} \psi$ with the given degrees holds since $\psi\left(U_{\beta}^{+}\right)=U_{\beta}^{+} K_{\beta}^{-1}$ and since $\varphi_{C}\left(I(C)_{-\beta}\right) \subseteq$ $U_{\beta}^{+} K_{\beta}^{-1}$ for all $\beta \in Q_{+}$. Moreover, $\phi_{C} \in \operatorname{Char}\left(U^{+}\left[w_{C}\right]\right)$ since $\varepsilon$ and $\varphi_{C}^{-1} \psi$ are algebra homomorphisms. Finally, let $\gamma \in Q$ and $x_{\gamma} \in U^{+}\left[w_{C}\right] \cap U_{\gamma}^{+}$. Then $y:=\varphi_{C}^{-1} \psi\left(x_{\gamma}\right) \in I(C)_{-\gamma}$. Hence, if $\phi_{C}\left(x_{\gamma}\right)=\varepsilon(y)=\operatorname{pr}_{(0,-\gamma)}(y) K_{\gamma} \neq 0$ then $\gamma \in L(C)^{\perp}$ by Lemma 2.7. Thus $L(C) \subseteq\left(\operatorname{supp} \phi_{C}\right)^{\perp}$.

(2) Lemma 2.13 implies that $D(w, \phi)$ is a connected right coideal subalgebra of $U \geq 0$. Moreover, $D(w, \phi)$ is ad $T_{L}$-stable since the adjoint action of $U^{0}$ commutes with $\psi$ and since $L \subseteq(\operatorname{supp} \phi)^{\perp}$. Hence $D(w, \phi) T_{L} \in \mathfrak{K}$ by Corollary 2.9.

(3) Let $C \in \mathfrak{K}$. For all $x \in \psi\left(U^{+}\left[w_{C}\right]\right)$ one obtains

$$
\phi_{C}\left(\psi^{-1}\left(x_{(1)}\right)\right) x_{(2)}=\varepsilon\left(\varphi_{C}^{-1}\left(x_{(1)}\right)\right) x_{(2)}=\varepsilon\left(\varphi_{C}^{-1}(x)_{(1)}\right) \varphi_{C}^{-1}(x)_{(2)}=\varphi_{C}^{-1}(x),
$$

where the second equation is satisfied by Proposition 2.10(1). Therefore $D\left(w_{C}, \phi_{C}\right)=$ $\varphi_{C}^{-1} \psi\left(U^{+}\left[w_{C}\right]\right)=I(C)$, and hence $D\left(w_{C}, \phi_{C}\right) T_{L(C)}=C$ by Proposition 2.8 (4).

Conversely, consider a triple $(w, \phi, L)$ contained in the codomain of the map in (3), and let $C=C(w, \phi, L)$. Then $I(C)=D(w, \phi)$ by Proposition 2.8(5) and $\varphi_{C}(D(w, \phi))=\psi\left(U^{+}[w]\right)$ by definition of $\varphi_{C}$ and $D(w, \varphi)$. Thus we have shown $w_{C}=w$ and $L(C)=L$. Further, one has

$$
\varphi_{C}\left(\phi\left(\psi^{-1}\left(y_{(1)}\right)\right) y_{(2)}\right)=y \quad \text { for all } y \in \psi\left(U^{+}[w]\right)
$$

by definition of $\varphi_{C}$. Let $x \in U^{+}[w]$ and let $y=\psi(x)$. Then

$$
\phi_{C}(x)=\varepsilon\left(\varphi_{C}^{-1} \psi(x)\right)=\varepsilon\left(\varphi_{C}^{-1}(y)\right)=\varepsilon\left(\phi\left(\psi^{-1}\left(y_{(1)}\right)\right) y_{(2)}\right)=\phi\left(\psi^{-1}(y)\right)=\phi(x),
$$

where the first equation follows from (2.14) and the third equation holds by (2.15). Hence we have $\phi_{C}=\phi$ which completes the proof of (3).

\section{Characters of $U^{+}[w]$}

Motivated by Theorem 2.15 we now turn to the classification of characters of $U^{+}[w]$. Throughout this section we fix an element $w \in W$ and a reduced expression

$$
w=s_{\alpha_{1}} \cdots s_{\alpha_{t}}, \quad \alpha_{1}, \ldots, \alpha_{t} \in \Pi
$$

of $w$ in terms of simple reflections, where $t=\ell(w)$. As in Section 2.1 for all $i=1, \ldots, t$ let $\beta_{i}=s_{\alpha_{1}} \cdots s_{\alpha_{i-1}} \alpha_{i}$. The set $\left\{\beta_{i} \mid i=1, \ldots, t\right\}$ coincides with

$$
\Phi_{w}^{+}:=\left\{\beta \in \Phi^{+} \mid w^{-1} \beta \in \Phi^{-}\right\} .
$$


Recall that $U^{+}[w]$ is the subalgebra of $U^{+}$generated by the root vectors $E_{\beta_{i}}:=$ $T_{\alpha_{1}} \cdots T_{\alpha_{i-1}} E_{\alpha_{i}}$ for $i=1, \ldots, t$. In the following, if we write $E_{\beta}$ for some $\beta \in \Phi_{w}^{+}$ we always mean the root vector corresponding to the fixed reduced expression (3.1) for the element $w$. If we write $\beta_{i}$ with a lower index $i=1, \ldots, t$ then we always refer to the specific ordering of $\Phi_{w}^{+}$from above.

3.1. Orthogonality. Let $\phi: U^{+}[w] \rightarrow k$ be a character. In the following lemma we show that roots corresponding to root vectors on which $\phi$ does not vanish, are mutually orthogonal. By the result of Levendorskil and Soibelman LS91, Proposition 5.5.2], Jan96, p. 164] one has for $i<j$ the commutation relation

$$
E_{\beta_{i}} E_{\beta_{j}}-q^{\left(\beta_{i}, \beta_{j}\right)} E_{\beta_{j}} E_{\beta_{i}}=\sum_{\left(a_{i+1}, \ldots, a_{j-1}\right) \in \mathbb{N}_{0}^{j-i-1}} m_{\left(a_{i+1}, \ldots, a_{j-1}\right)} E_{\beta_{i+1}}^{a_{i+1}} \cdots E_{\beta_{j-1}}^{a_{j-1}} .
$$

for some coefficients $m_{\left(a_{i+1}, \ldots, a_{j-1}\right)} \in k$. These commutation relations are the main ingredient to obtain the following result.

Lemma 3.1. Let $\phi: U^{+}[w] \rightarrow k$ be a character and $\left(\beta_{i}, \beta_{j}\right) \neq 0$ for some $i \neq j$. Then $\phi\left(E_{\beta_{i}}\right)=0$ or $\phi\left(E_{\beta_{j}}\right)=0$.

Proof. Consider Equation (3.2) for $i<j$. If the right hand side is zero, then the claim of the lemma holds. We now perform an indirect proof. Assume that $j-i>0$ is minimal such that $\left(\beta_{i}, \beta_{j}\right) \neq 0$ and such that $\phi\left(E_{\beta_{i}}\right) \neq 0$ and $\phi\left(E_{\beta_{j}}\right) \neq 0$. Then the right hand side of Equation (3.2) is nonzero and contains a monomial $E_{\beta_{i+1}}^{a_{i+1}} \cdots E_{\beta_{j-1}}^{a_{j-1}}$ such that $\phi\left(E_{\beta_{m}}\right) \neq 0$ whenever $i<m<j$ and $a_{m} \neq 0$. Thus by Lemma A.2 we can find an $n$ with $i<n<j$ such that $\phi\left(E_{\beta_{n}}\right) \neq 0$ and one of $\left(\beta_{i}, \beta_{n}\right),\left(\beta_{j}, \beta_{n}\right)$ is nonzero. This is a contradiction to the minimality of $j-i>0$.

For any $\phi \in \operatorname{Char}\left(U^{+}[w]\right)$ define

$$
\Phi_{w}^{+}(\phi):=\left\{\beta \in \Phi_{w}^{+} \mid \phi\left(E_{\beta}\right) \neq 0\right\} .
$$

We will see in Remark 3.4 that $\Phi_{w}^{+}(\phi)$ is independent of the chosen reduced expression for $w$.

Remark 3.2. By Lemma 3.1 for any $\phi \in \operatorname{Char}\left(U^{+}[w]\right)$ the set $\Phi_{w}^{+}(\phi)$ consists of pairwise orthogonal roots. In particular, $\Phi_{w}^{+}(\phi)$ is linearly independent and contains at most $\operatorname{rank}(\mathfrak{g})$ elements.

3.2. Polynomial $H$-prime ideals. The algebra $U^{+}[w]$ is $Q$-graded via the direct sum decomposition $U^{+}[w]=\oplus_{\alpha \in Q}\left(U^{+}[w] \cap U_{\alpha}^{+}\right)$. Equivalently, $U^{+}[w]$ has a rational action of the torus $H:=\left(k^{\times}\right)^{\operatorname{rank}(\mathfrak{g})}$ by $k$-algebra automorphisms, where $k^{\times}=k \backslash\{0\}$, (cf. [BG02, II.2.11]). This will allow us to follow BG02, Chapter II] and apply the theory of prime ideals invariant under a torus action to determine $\operatorname{Char}\left(U^{+}[w]\right)$. Recall that a proper ideal $P$ of an associative $k$-algebra $A$ is called prime if any two ideals $I, J$ not contained in $P$ satisfy $I J \nsubseteq P$. Equivalently, $P$ is prime if for all $a, b \in A \backslash P$ there exists $c \in A$ with $a c b \notin P$. In particular, if $P$ is prime and $A / P$ is commutative, then $a, b \in A \backslash P$ implies that $a b \notin P$. If $A$ admits an action of the torus $H$ by automorphisms, then an ideal $P$ of $A$ is called $H$-prime, see [BG02, II.1.9], if any two ideals $I, J$ stable under the action of $H$ and not contained in $P$ satisfy $I J \nsubseteq P$. If, moreover, $A$ is noetherian and $H$ acts rationally, then $H$-prime ideals are prime [BG02, II.2.9]. All these assumptions are fulfilled for the action of $H=\left(k^{\times}\right)^{\text {rankg }}$ on $U^{+}[w]$. 
Let $\phi: U^{+}[w] \rightarrow k$ be a character. Then $\operatorname{ker}(\phi)$ is an ideal in $U^{+}[w]$ which is one-codimensional and hence prime. The intersection

$$
P_{\phi}:=\bigcap_{h \in H} h \operatorname{ker}(\phi)
$$

is an $H$-prime ideal of $U^{+}[w]$. Indeed, let $I$ and $J$ be $H$-stable (or equivalently $Q$ graded) ideals such that $I J \subseteq P_{\phi} \subseteq \operatorname{ker}(\phi)$. Then, say, $I \subseteq \operatorname{ker}(\phi)$ because $\operatorname{ker}(\phi)$ is prime and hence $I \subseteq P_{\phi}$ since $I$ is $H$-stable. Following [BG02, II.1.9] the set of $H$-prime ideals in $U^{+}[w]$ will be denoted by $H-\operatorname{spec}\left(U^{+}[w]\right)$. The $H$-prime ideals of $U^{+}[w]$ obtained in the above way via characters have very special properties as illustrated by the following lemma.

Proposition 3.3. Let $\phi: U^{+}[w] \rightarrow k$ be a character and $P_{\phi}$ the corresponding $H$-prime ideal defined by Equation (3.4). Then the quotient $U^{+}[w] / P_{\phi}$ is a commutative polynomial ring in the root vectors $E_{\beta}$ with $\beta \in \Phi_{w}^{+}(\phi)$.

Proof. The quotient algebra $U^{+}[w] / P_{\phi}$ is generated by the root vectors $E_{\beta}$ with $\beta \in \Phi_{w}^{+}(\phi)$. Assume now that $\phi\left(E_{\beta_{i}}\right) \neq 0$ and $\phi\left(E_{\beta_{j}}\right) \neq 0$ for some $i<j$. We know from Lemma 3.1 that $\left(\beta_{i}, \beta_{j}\right)=0$. Hence by Equation (3.2) the commutator of $E_{\beta_{i}}$ and $E_{\beta_{j}}$ is a polynomial in the $E_{\beta_{m}}$ of weight $\beta_{i}+\beta_{j}$. As $\left(\beta_{i}, \beta_{i}+\beta_{j}\right)=\left(\beta_{i}, \beta_{i}\right) \neq 0$ each monomial in this polynomial contains a root vector $E_{\beta_{m}}$ with $m \neq i$ and $\left(\beta_{i}, \beta_{m}\right) \neq 0$. By Lemma 3.1 this implies $\phi\left(E_{\beta_{m}}\right)=0$ and hence $E_{\beta_{m}} \in P_{\phi}$. Thus, the right hand side of Equation (3.2) is contained in $P_{\phi}$ and the commutator $\left[E_{\beta_{i}}, E_{\beta_{j}}\right]$ is zero in $U^{+}[w] / P_{\phi}$. This proves that $U^{+}[w] / P_{\phi}$ is commutative.

It remains to show that in $U^{+}[w] / P_{\phi}$ there are no relations between the $E_{\beta}$ with $\beta \in \Phi_{w}^{+}(\phi)$ apart from commutativity. To this end assume that $P_{\phi}$ contains a nonzero commutative polynomial in the $E_{\beta}$ with $\beta \in \Phi_{w}^{+}(\phi)$. By linear independence of the set $\Phi_{w}^{+}(\phi)$ observed in Remark 3.2 one can use the torus action (or equivalently the $Q$-grading) to show that $P_{\phi}$ already contains a monomial in the $E_{\beta}$ with $\beta \in \Phi_{w}^{+}(\phi)$. As noted above $P_{\phi}$ is a prime ideal and $U^{+}[w] / P_{\phi}$ is commutative. Hence one gets the contradiction $E_{\beta} \in P_{\phi}$ for some $\beta \in \Phi_{w}^{+}(\phi)$.

Remark 3.4. By Proposition 3.3 the set $\Phi_{w}^{+}(\phi)$ defined in (3.3) is uniquely determined by the $H$-prime ideal $P_{\phi}$. Hence $\Phi_{w}^{+}(\phi)$ only depends on the character $\phi$ and not on the chosen reduced expression for $w$.

Proposition 3.3 motivates the following definition.

Definition 3.5. An $H$-prime ideal $P$ of $U^{+}[w]$ is called polynomial if $U^{+}[w] / P$ is a commutative polynomial ring in the $E_{\beta}, \beta \in \Phi_{w}^{+}$, which are not contained in $P$. The set of polynomial $H$-prime ideals in $U^{+}[w]$ is denoted by $H-\operatorname{spec}^{\text {poly }}\left(U^{+}[w]\right)$.

Lemma 3.6. Let $P \in H-\operatorname{spec}^{\text {poly }}\left(U^{+}[w]\right)$. The ideal $P$ is generated by the set $\left\{E_{\beta} \mid \beta \in \Phi_{w}^{+}, E_{\beta} \in P\right\}$.

Proof. This follows from the PBW-Theorem for $U^{+}[w]$, cf. Jan96, 8.24].

To summarize results we collect some equivalent characterizations of polynomial $H$-prime ideals of $U^{+}[w]$.

Proposition 3.7. For any $H$-prime ideal $P$ of $U^{+}[w]$ the following are equivalent:

(1) The $H$-prime ideal $P$ is polynomial.

(2) The algebra $U^{+}[w] / P$ is commutative. 
(3) There exists $\phi \in \operatorname{Char}\left(U^{+}[w]\right)$ such that the prime ideal $P$ is generated by the set $\left\{E_{\beta} \mid \beta \in \Phi_{w}^{+} \backslash \Phi_{w}^{+}(\phi)\right\}$.

Proof. The equivalence of properties (1) and (3) is immediate from Lemma 3.6 and Proposition 3.3. It remains to show that (2) implies (1). To this end assume that $P$ is an $H$-prime ideal such that $U^{+}[w] / P$ is commutative and define

$$
J=\left\{i \in\{1, \ldots, t\} \mid E_{\beta_{i}} \notin P\right\} .
$$

We claim that $\left(\beta_{i}, \beta_{j}\right)=0$ for all distinct $i, j \in J$. Indeed, otherwise choose elements $i<j$ in $J$ with $j-i$ minimal such that $\left(\beta_{i}, \beta_{j}\right) \neq 0$ and $E_{\beta_{i}}, E_{\beta_{j}} \notin$ $P$. This implies for any $m$ with $i<m<j$ that $E_{\beta_{m}} \in P$ if $\left(\beta_{i}, \beta_{m}\right) \neq 0$ or $\left(\beta_{m}, \beta_{j}\right) \neq 0$. Hence by Lemma A.2 the right hand side of Equation (3.2) belongs to $P$ for the chosen $i, j$. The assumption $\left(\beta_{i}, \beta_{j}\right) \neq 0$ now implies that $E_{\beta_{i}} E_{\beta_{j}} \in P$. But $U^{+}[w] / P$ is commutative and $P$ is prime, which gives the contradiction to $E_{\beta_{i}}, E_{\beta_{j}} \notin P$. This proves that indeed $\left(\beta_{i}, \beta_{j}\right)=0$ for all distinct $i, j \in J$.

Assume now that a commutative polynomial $f$ in the $E_{\beta_{i}}, i \in J$, belongs to $P$. Using the $H$-stability of $P$ and the orthogonality one may assume that $f$ is a monomial. Yet this yields again a contradiction to the fact that $P$ is prime and $U^{+}[w] / P$ is commutative.

3.3. Stratification of $\operatorname{Char}\left(U^{+}[w]\right)$. By Proposition 3.3 one has a surjective map

$$
\operatorname{pr}_{w}: \operatorname{Char}\left(U^{+}[w]\right) \rightarrow H-\operatorname{spec}^{\text {poly }}\left(U^{+}[w]\right), \quad \phi \mapsto \operatorname{pr}_{w}(\phi):=P_{\phi} .
$$

For each $P \in H-\operatorname{spec}^{p o l y}\left(U^{+}[w]\right)$ the preimage $\operatorname{pr}_{w}^{-1}(P)$ is isomorphic to the spectrum of a Laurent polynomial ring in as many variables as there are elements in $\left\{\beta \in \Phi_{w}^{+} \mid E_{\beta} \notin P\right\}$. Therefore the set $\operatorname{Char}\left(U^{+}[w]\right)$ is a disjoint union

$$
\operatorname{Char}\left(U^{+}[w]\right)=\underset{P \in H-\operatorname{spec}^{p o l y}\left(U^{+}[w]\right)}{\operatorname{pr}_{w}^{-1}(P)}
$$

of spectra of Laurent polynomial rings. The decomposition (3.5) is the $H$-stratification of $\operatorname{Char}\left(U^{+}[w]\right)$ in the sense of $[\mathrm{BG} 02$, II.2.1]. In order to classify characters of $U^{+}[w]$ it hence remains to determine all polynomial $H$-prime ideals $P$ of $U^{+}[w]$ and for each of them the set $\left\{\beta \in \Phi_{w}^{+} \mid E_{\beta} \notin P\right\}$.

Let $\Theta \subseteq \Phi^{+}$be a set of pairwise orthogonal roots. Then the reflections $s_{\beta}$ and $s_{\gamma}$ commute for any $\beta, \gamma \in \Theta$. Hence we may write $\Pi_{\beta \in \Theta} s_{\beta}$ to denote the product of all reflections corresponding to roots in $\Theta$. Let

$$
w_{\Theta}=\left(\Pi_{\beta \in \Theta} s_{\beta}\right) w
$$

and define

$$
\begin{gathered}
T^{w}=\left\{\Theta \subseteq \Phi_{w}^{+} \mid \Theta\right. \text { consists of pairwise orthogonal roots, } \\
\text { and } \left.\ell\left(w_{\Theta}\right)=\ell(w)-|\Theta|\right\} .
\end{gathered}
$$

For any $\Theta \in T^{w}$ and any $\beta \in \Phi_{w}^{+}$one has the implication

$$
\beta \in \Theta \Longrightarrow w_{\Theta}^{-1} \beta \in \Phi^{+} .
$$

In explicit examples of small rank the set $T^{w}$ is not hard to determine. Let $J \subseteq$ $\{1, \ldots, t\}$ be a subset such that the elements of $\Theta:=\left\{\beta_{i} \mid i \in J\right\}$ are pairwise orthogonal. Then the set $\Theta$ belongs to $T^{w}$ if and only if one obtains a reduced expression by deleting all simple reflections $s_{\alpha_{i}}$ for $i \in J$ from the expression (3.1) 
for $w$. Moreover, by the following lemma, all sets in $T^{w}$ can be built by an inductive procedure from smaller sets in $T^{w}$.

Lemma 3.8. Let $w \in W$ and $\Theta \in T^{w}$. If $\Theta^{\prime} \subseteq \Theta$ then $\Theta^{\prime} \in T^{w}$.

Proof. Let $\Theta^{\prime \prime} \subset \Theta$ and $\beta \in \Theta \backslash \Theta^{\prime \prime}$. For any weight $\lambda$ the orthogonality assumption implies that $\left(\beta, w_{\Theta^{\prime \prime}} \lambda\right)=(\beta, w \lambda)$. Hence, if $\lambda$ is regular dominant, that is $(\alpha, \lambda)>0$ for all $\alpha \in \Pi$, then $\left(\beta, w_{\Theta^{\prime \prime}} \lambda\right)<0$ because $w^{-1} \beta<0$. By Lemma A.3 this implies $s_{\beta} w_{\Theta^{\prime \prime}}<w_{\Theta^{\prime \prime}}$. Repeated application of this argument leads to

$$
\Theta^{\prime} \varsubsetneqq \Theta^{\prime \prime} \subseteq \Theta \quad \Longrightarrow \quad w_{\Theta} \leq w_{\Theta^{\prime \prime}}<w_{\Theta^{\prime}} .
$$

Together with $l\left(w_{\Theta}\right)=l(w)-|\Theta|$ this implies $l\left(w_{\Theta^{\prime}}\right)=l(w)-\left|\Theta^{\prime}\right|$ for any $\Theta^{\prime} \subseteq \Theta$. Hence any subset $\Theta^{\prime} \subseteq \Theta$ belongs to $T^{w}$.

The set $T^{w}$ will provide us with the desired parametrization of $H-\operatorname{spec}^{\text {poly }}\left(U^{+}[w]\right)$. As a first step we associate a polynomial $H$-prime ideal to each element in $T^{w}$.

Proposition 3.9. Let $\Theta \in T^{w}$ and let $P_{\Theta}$ denote the two-sided ideal of $U^{+}[w]$ generated by all $E_{\beta}$ with $\beta \in \Phi_{w}^{+} \backslash \Theta$. Then $P_{\Theta}$ is a polynomial $H$-prime ideal of $U^{+}[w]$, and $E_{\beta} \notin P_{\Theta}$ for all $\beta \in \Theta$.

Proof. We may assume that $\mathfrak{g}$ is simple. By [Jan96, 8.24] the algebra $U^{+}[w]$ can be given in terms of generators $E_{\beta_{i}}, i=1, \ldots, t$, and relations (3.2). It suffices to show that for any $m, n \in\{1, \ldots, t\}$ with $m<n$ there is no family $\left\{a_{j} \in \mathbb{N}_{0}\right\}_{\beta_{j} \in \Theta}$ such that

$$
\beta_{m}+\beta_{n}=\sum_{\beta_{j} \in \Theta, m<j<n} a_{j} \beta_{j} .
$$

Indeed, if no such family exists, then any nontrivial monomial on the right hand side of Equation (3.2) contains a factor in $P_{\Theta}$.

If $\mathfrak{g}$ is of rank 2 then $T^{w}$ consists only of subsets of $\left\{\beta_{1}, \beta_{t}\right\}$. In this case the right hand side of Equation (3.9) always vanishes. Hence we may assume that $\operatorname{rank}(\mathfrak{g}) \geq 3$.

We prove the impossibility of (3.9) indirectly. Assume we have found a family $\left\{a_{j} \in \mathbb{N}_{0}\right\}_{\beta_{j} \in \Theta}$ such that (3.9) holds. By Lemma 3.8 all subsets of $\Theta$ are also contained in $T^{w}$. Thus, leaving out certain elements of $\Theta$ we may assume that all $a_{j}$ are strictly positive and that $\beta_{m}, \beta_{n} \notin \Theta$. Moreover, shortening $w$ from both sides if necessary, we may assume that $m=1$ and $n=t$. Hence we have

$$
\alpha_{1}+\beta_{t}=\sum_{\beta_{j} \in \Theta} a_{j} \beta_{j} .
$$

Now let $\beta_{t}^{\prime}=-w_{\Theta} \alpha_{t} \in \Phi^{+}$. The assumptions $\Theta \in T^{w}$ and $\alpha_{1} \notin \Theta$ imply that

$$
w_{\Theta}^{-1} \alpha_{1} \in \Phi^{-}, \quad w_{\Theta}^{-1} \beta_{t}^{\prime}=-\alpha_{t} \in \Phi^{-} .
$$

Using the definition (3.6) of $w_{\Theta}$ and the orthogonality of the $\beta_{j} \in \Theta$ one calculates

$$
\beta_{t}^{\prime}=\beta_{t}-2 \sum_{\beta_{j} \in \Theta} \frac{\left(\beta_{j}, \beta_{t}\right)}{\left(\beta_{j}, \beta_{j}\right)} \beta_{j} .
$$

Hence one obtains that

$$
\alpha_{1}+\beta_{t}^{\prime}=\sum_{\beta_{j} \in \Theta}\left(a_{j}-2 \frac{\left(\beta_{j}, \beta_{t}\right)}{\left(\beta_{j}, \beta_{j}\right)}\right) \beta_{j} .
$$


Apply $w_{\Theta}^{-1}$ to this equation. By (3.11) and since $w_{\Theta}^{-1} \beta_{j} \in \Phi^{+}$for all $\beta_{j} \in \Theta$ by (3.8), we conclude that there exists $\beta_{i} \in \Theta$ such that

$$
0<a_{i}<\frac{2\left(\beta_{i}, \beta_{t}\right)}{\left(\beta_{i}, \beta_{i}\right)}
$$

Hence $\beta_{t}$ is long by Lemma A.1 Replacing $w$ by $w^{-1}$ and $\Theta$ by $-w^{-1}(\Theta)$ and applying the same procedure one obtains that $\alpha_{1}$ has to be long. Again by LemmaA.1 the root $\beta_{i} \in \Theta$ satisfying (3.13) has to be short. Then $\left(\alpha_{1}, \beta_{i}\right),\left(\beta_{i}, \beta_{t}\right) \in\{2,-2\}$, since $\mathfrak{g}$ is irreducible and not of type $G_{2}$. Further, $\left(\alpha_{1}, \beta_{i}\right)+\left(\beta_{t}, \beta_{i}\right)=2 a_{i}>0$ by Equation (3.10), and hence $\left(\alpha_{1}, \beta_{i}\right)=\left(\beta_{t}, \beta_{i}\right)=2$. This is a contradiction to Relation (3.13).

Remark 3.10. We will see in Corollary 3.16 that for any $\Theta \in T^{w}$ the polynomial $H$-prime ideal $P_{\Theta}$ obtained by Proposition 3.9 is independent of the chosen reduced expression for $w$.

3.4. $H$-prime ideals with noncommutative quotients. The next aim is to show that Proposition 3.9 already produces as many distinct polynomial $H$-prime ideals as we can possibly find. To this end we resort to the description of $\mathrm{H}-$ $\operatorname{spec}\left(U^{+}[w]\right)$ recently given by Yakimov [Yak09] based on results by Gorelik Gor00. In order to refer to these papers without much rewriting, we work with $U^{-}[w]$ instead of $U^{+}[w]$ for now. Recall from [Jan96, 8.24] that by definition $U^{-}[w]=$ $\omega\left(U^{+}[w]\right)$ where $\omega$ denotes the algebra automorphism of $U$ defined by

$$
\omega\left(E_{\alpha}\right)=F_{\alpha}, \quad \omega\left(F_{\alpha}\right)=E_{\alpha}, \quad \omega\left(K_{\alpha}\right)=K_{\alpha}^{-1} \quad \text { for all } \alpha \in \Pi .
$$

Let $\Lambda$ denote the weight lattice and $\Lambda^{+}$the set of dominant weights. In this subsection we mostly use Gorelik's and Joseph's notation following Gor00, Jos95. For any dominant integral weight $\lambda \in \Lambda^{+}$let $V(\lambda)$ denote the corresponding simple $U$-module and let $v_{\lambda} \in V(\lambda)$ be a highest weight vector. We write $V(\lambda)^{*}$ to denote the dual space of $V(\lambda)$ which is canonically a right $U_{q}(\mathfrak{g})$-module. Let $k_{q}[G]$ be the Hopf algebra generated by all matrix coefficients $c_{f, v}^{\lambda}$ for $v \in V(\lambda), f \in V(\lambda)^{*}$ of the representations $V(\lambda), \lambda \in \Lambda^{+}$. We define

$$
R^{+}=\bigoplus_{\lambda \in \Lambda^{+}} V(\lambda)^{*}
$$

which is an algebra with the Cartan multiplication. We can consider $R^{+}$as a subalgebra of $k_{q}[G]$ if we identify $f \in V(\lambda)^{*}$ with the matrix coefficient $c_{f, v_{\lambda}}^{\lambda}$. Let $\xi_{w \lambda} \in V(\lambda)^{*}$ be a functional which only lives on the weight space of weight $w \lambda$ in $V(\lambda)$. To shorten notation we write $c_{\xi_{w \lambda}, v_{\lambda}}^{\lambda}$ simply as $c_{w}^{\lambda}$. The elements $c_{w}^{\lambda}$ are defined up to scalars. By [Jos95, 9.1.10] these scalars can be chosen such that $c_{w}^{\mu} c_{w}^{\nu}=c_{w}^{\mu+\nu}$ for any $\mu, \nu \in \Lambda^{+}$and $c_{w}:=\left\{c_{w}^{\lambda} \mid \lambda \in \Lambda^{+}\right\}$becomes an Ore set in $R^{+}$. Recall that we have fixed $w$ for all of this section, however, we can define $c_{y}^{\lambda}$ and $c_{y}$ analogously for any $y \in W$. Consider the localized algebra $R^{w}:=R\left[c_{w}^{-1}\right]$. This algebra is $\Lambda$-graded with $\operatorname{deg}\left(\left(c_{w}^{\mu}\right)^{-1} c_{f, v_{\nu}}^{\nu}\right)=\nu-\mu$ for all $\mu, \nu \in \Lambda^{+}, f \in V(\nu)^{*}$. Let $R_{0}^{w}$ denote the algebra of elements of degree zero in $R^{w}$. Using the abbreviation $c_{w}^{-\lambda}:=\left(c_{w}^{\lambda}\right)^{-1}$ one may write

$$
R_{0}^{w}=\sum_{\lambda \in \Lambda^{+}} c_{w}^{-\lambda} V(\lambda)^{*}=\underset{\lambda \in \Lambda^{+}}{\lim _{w}} c_{w}^{-\lambda} V(\lambda)^{*} .
$$


In particular, any element of $R_{0}^{w}$ can be written in the form $c_{w}^{-\lambda} c_{f, v_{\lambda}}^{\lambda}$ for some $\lambda \in \Lambda^{+}$and $f \in V(\lambda)^{*}$.

Following [Gor00, 5.2.3, 6.1.2], for any $y \in W$ define ideals $Q(y)^{ \pm}$of $R^{+}$and $Q(y)_{w}^{ \pm}$of $R_{0}^{w}$ by

$$
\begin{aligned}
& Q(y)^{ \pm}=\sum_{\lambda \in \Lambda^{+}}\left\{c_{\xi, v_{\lambda}}^{\lambda} \mid \xi \in V(\lambda)^{*}, \xi \perp U^{ \pm} v_{y \lambda}\right\}, \\
& Q(y)_{w}^{ \pm}=\underset{\lambda \in \Lambda^{+}}{\lim _{w}}\left\{c_{w}^{-\lambda} c_{\xi, v_{\lambda}}^{\lambda} \mid \xi \in V(\lambda)^{*}, \xi \perp U^{ \pm} v_{y \lambda}\right\},
\end{aligned}
$$

where $v_{y \lambda} \in V(\lambda)$ is a weight vector of weight $y \lambda$. (For the fact that $Q(y)^{ \pm}$ and $Q(y)_{w}^{ \pm}$are ideals confer to Gorelik's reference to [Jos95, 10.1.8]. Consult also Yak09, 3.1].) By definition, for any $y, y^{\prime} \in W$ with $y \leq y^{\prime}$ one obtains the inclusions $Q\left(y^{\prime}\right)_{w}^{+} \subseteq Q(y)_{w}^{+}$and $Q(y)_{w}^{-} \subseteq Q\left(y^{\prime}\right)_{w}^{-}$.

For our purposes it is sufficient to consider for any $y \leq w$ the subspaces

$$
\begin{aligned}
Q(y, w)_{w} & :=Q(y)_{w}^{-}+Q(w)_{w}^{+} \subset R_{0}^{w}, \\
Q(y, w) & :=\left\{a \in R^{+} \mid \exists \lambda \in \Lambda^{+}: c_{w}^{\lambda} a \in Q(y)^{-}+Q(w)^{+}\right\} \subset R^{+} .
\end{aligned}
$$

For any $y \leq w$ the subspace $Q(y, w)_{w}$ is an $H$-stable prime ideal of $R_{0}^{w}$ by Gor00, 6.6], and $Q(y, w)$ is an $H$-stable prime ideal of $R^{+}$by [Gor00, 6.7].

Lemma 3.11. Gor00, Lemma 6.10] Let $y, y^{\prime} \in W$ with $y \leq y^{\prime} \leq w$. Then $Q(y, w) \cap c_{y^{\prime}}=\emptyset$.

Corollary 3.12. Let $\lambda \in \Lambda^{+}$and $y, y^{\prime} \in W$ with $y \leq y^{\prime} \leq w$. Then $c_{w}^{-\lambda} c_{y^{\prime}}^{\lambda}$ is not contained in $Q(y, w)_{w}$.

Proof. Assume that $c_{w}^{-\lambda} c_{y^{\prime}}^{\lambda} \in Q(y, w)_{w}$ for some $y \leq y^{\prime} \leq w$. Then there exists $\mu \in \Lambda^{+}$such that

$$
c_{w}^{-\lambda} c_{y^{\prime}}^{\lambda}=c_{w}^{-\mu} c_{\xi, v_{\mu}}^{\mu}+c_{w}^{-\mu} c_{\zeta, v_{\mu}}^{\mu}
$$

for some $\xi \in\left(U^{-} v_{y \mu}\right)^{\perp}$ and $\zeta \in\left(U^{+} v_{w \mu}\right)^{\perp}$. Multiplying from the left by $c_{w}^{\lambda+\mu}$ and setting $\eta=\lambda+\mu$ one obtains (using that $Q(y)^{-}$and $Q(w)^{+}$are ideals) that

$$
c_{w}^{\mu} c_{y^{\prime}}^{\lambda}=c_{\xi^{\prime}, v_{\eta}}^{\eta}+c_{\zeta^{\prime}, v_{\eta}}^{\eta}
$$

for some $\xi^{\prime} \in\left(U^{-} v_{y \eta}\right)^{\perp}$ and $\zeta^{\prime} \in\left(U^{+} v_{w \eta}\right)^{\perp}$. By definition of $Q(y, w)$ this means that $c_{y^{\prime}}^{\lambda} \in Q(y, w)$ which is a contradiction to Lemma 3.11

The following proposition is the main technical tool to show that certain $H$-prime ideals of $U^{+}[w]$ are not polynomial.

Proposition 3.13. Let $\beta, \gamma \in \Phi^{+}$. Define $y^{\prime}=s_{\gamma} w$ and $y=s_{\beta} s_{\gamma} w$ and assume that $y<y^{\prime}<w$. Then in $R_{0}^{w} / Q(y, w)_{w}$ the relation

$$
\left(c_{w}^{-\lambda} c_{y}^{\lambda}\right)\left(c_{w}^{-\lambda} c_{y^{\prime}}^{\lambda}\right)=q^{C(\lambda)}\left(c_{w}^{-\lambda} c_{y^{\prime}}^{\lambda}\right)\left(c_{w}^{-\lambda} c_{y}^{\lambda}\right)
$$

holds for all $\lambda \in \Lambda^{+}$, where $C(\lambda)=\left(w \lambda-y^{\prime} \lambda, y \lambda-y^{\prime} \lambda\right)$. In particular, if $(\beta, \gamma) \neq 0$ then $R_{0}^{w} / Q(y, w)_{w}$ is not commutative.

Proof. Following [Gor00, Section 4] we use commutation relations in $k_{q}[G]$ which appeared as standard tools for instance in [Jos95, 9.1]. For any $\eta \in \Lambda$ define $J_{\lambda}^{+}(\eta)_{w}$ (resp. $\left.J_{\lambda}^{-}(\eta)_{w}\right)$ to be the left ideal of $R_{0}^{w}$ generated by $c_{w}^{-\lambda} c_{f_{\eta^{\prime}}, v_{\lambda}}^{\lambda}$ with $\eta^{\prime}<\eta$ (resp. 
$\left.\eta^{\prime}>\eta\right)$, where $f_{\eta^{\prime}} \in V(\lambda)^{*}$ only lives on the weight space of weight $\eta^{\prime}$ in $V(\lambda)$. By definition one has for all $w^{\prime} \in W$ and $\lambda \in \Lambda^{+}$the inclusions

$$
J_{\lambda}^{+}\left(w^{\prime} \lambda\right)_{w} \subseteq Q\left(w^{\prime}\right)_{w}^{+}, \quad J_{\lambda}^{-}\left(w^{\prime} \lambda\right)_{w} \subseteq Q\left(w^{\prime}\right)_{w}^{-}
$$

and hence definition (3.15) gives

$$
J_{\lambda}^{+}(w \lambda)_{w}+J_{\lambda}^{-}(y \lambda)_{w} \subseteq Q(y, w)_{w} .
$$

By [Gor00, Lemma 4.2(i)] one has

$$
c_{w}^{-2 \lambda} c_{y^{\prime}}^{\lambda} c_{w}^{\lambda}=q^{(\lambda, \lambda)-\left(w \lambda, y^{\prime} \lambda\right)} c_{w}^{-\lambda} c_{y^{\prime}}^{\lambda} \bmod J_{\lambda}^{+}(w \lambda)_{w}
$$

and by [Gor00, Lemma 4.4(iv)] one has

$$
\left(c_{w}^{-\lambda} c_{y}^{\lambda}\right)\left(c_{w}^{-\lambda} c_{y^{\prime}}^{\lambda}\right)=q^{-\left(y \lambda, y^{\prime} \lambda-w \lambda\right)}\left(c_{w}^{-2 \lambda} c_{y^{\prime}}^{\lambda} c_{w}^{\lambda}\right)\left(c_{w}^{-\lambda} c_{y}^{\lambda}\right) \quad \bmod J_{\lambda}^{-}(y \lambda)_{w} .
$$

The inclusion (3.18) together with Equations (3.19) and (3.20) imply the relation

$$
\left(c_{w}^{-\lambda} c_{y}^{\lambda}\right)\left(c_{w}^{-\lambda} c_{y^{\prime}}^{\lambda}\right)=q^{C(\lambda)}\left(c_{w}^{-\lambda} c_{y^{\prime}}^{\lambda}\right)\left(c_{w}^{-\lambda} c_{y}^{\lambda}\right) \bmod Q(y, w)_{w}
$$

with $C(\lambda)=-\left(y \lambda, y^{\prime} \lambda-w \lambda\right)+(\lambda, \lambda)-\left(w \lambda, y^{\prime} \lambda\right)=\left(w \lambda-y^{\prime} \lambda, y \lambda-y^{\prime} \lambda\right)$. This proves the commutation relation (3.17).

To prove the second statement recall from Corollary 3.12 that both $c_{w}^{-\lambda} c_{y}^{\lambda}$ and $c_{w}^{-\lambda} c_{y^{\prime}}^{\lambda}$ are nonzero elements in $R_{0}^{w} / Q(y, w)_{w}$. We now assume that $\lambda$ is a regular weight, i.e. that the Weyl group acts faithfully on $\lambda$. Then $w \lambda-y^{\prime} \lambda$ is a nonzero multiple of $\gamma$, and similarly $y^{\prime} \lambda-y \lambda$ is a nonzero multiple of $\beta$. Hence $C(\lambda)$ is a nonzero multiple of $(\beta, \gamma)$ and therefore nonzero by assumption. Since $Q(y, w)_{w}$ is prime, we conclude that $R_{0}^{w} / Q(y, w)_{w}$ is not commutative.

3.5. Classification of characters. By [Yak09, Theorem 3.8] there exists an order preserving bijection between the poset of $H$-prime ideals of $U^{-}[w]$ ordered by inclusion and the set

$$
W^{\leq w}:=\{y \in W \mid y \leq w\}
$$

with the Bruhat order. Let $P^{-}(y)$ denote the $H$-prime ideal of $U^{-}[w]$ corresponding to $y \in W \leq w$. More explicitly, Yakimov constructs a surjective algebra homomorphism

$$
\phi_{w}: R_{0}^{w} \rightarrow U^{-}[w]
$$

with kernel $Q(w)_{w}^{+}$which is moreover compatible with the $H$-action Yak09, Proposition 3.6]. The $H$-prime ideals of $U^{-}[w]$ are hence in one-to-one correspondence to the $H$-prime ideals of $R_{0}^{w}$ which contain $Q(w)_{w}^{+}$. These are known to be of the form $Q(y, w)_{w}$ for all $y \in W^{\leq w}$ by Gor00] (cf. [Yak09, Theorem 3.1]). By definition $P^{-}(y)=\phi_{w}\left(Q(y, w)_{w}\right)$ and hence

$$
U^{-}[w] / P^{-}(y) \cong R_{0}^{w} / Q(y, w)_{w} .
$$

Recall that $\omega\left(U^{-}[w]\right)=U^{+}[w]$ where $\omega$ denotes the involutive algebra isomorphism defined by (3.14). For any $y \leq w$ let $P^{+}(y)=\omega\left(P^{-}(y)\right)$. Then $P^{+}(y)$ is an $H$ prime ideal of $U^{+}[w]$ since $\omega$ is an algebra isomorphism and $\omega\left(U^{0}\right)=U^{0}$. By the results for $U^{-}[w]$ explained above, any $H$-prime ideal of $U^{+}[w]$ is of the form $P^{+}(y)$ for some $y \in W \leq w$. Moreover, $\omega$ induces an algebra isomorphism

$$
U^{-}[w] / P^{-}(y) \cong U^{+}[w] / P^{+}(y) .
$$


Recall the definition of $T^{w}$ from (3.7). To identify the set of polynomial $H$-prime ideals of $U^{+}[w]$ with a subset of $W^{\leq w}$ define

$$
W^{w}=\left\{w_{\Theta} \mid \Theta \in T^{w}\right\} .
$$

By Lemma 3.8 one has $W^{w} \subseteq W \leq w$. Define a map

$$
\varkappa^{w}: T^{w} \rightarrow W^{w}, \quad \varkappa^{w}(\Theta)=w_{\Theta}
$$

The set $T^{w}$ is partially ordered by inclusion while the set $W^{w}$ is partially ordered by the Bruhat order on $W$.

Lemma 3.14. The map $\varkappa^{w}$ is an order reversing bijection.

Proof. The map $\varkappa^{w}$ is surjective by definition and order reversing by Lemma 3.8 It follows from [BB06, Lemma 2.7.2] that for any $\Theta \in T^{w}$ there exists at most one sequence $j_{1}<j_{2}<\cdots<j_{|\Theta|}$ such that the elements $w_{m}:=s_{j_{m}} s_{j_{m+1}} \cdots s_{j_{|\Theta|}} w$ satisfy

$$
w_{\Theta}=w_{1}<w_{2}<\cdots<w_{|\Theta|}<w .
$$

Hence $\varkappa^{w}$ is injective by Lemma 3.8 and the fact that the reflections $s_{\beta}$ for $\beta \in \Theta$ commute for any $\Theta \in T^{w}$.

Proposition 3.15. Let $y \in W^{\leq w} \backslash W^{w}$. Then $U^{+}[w] / P^{+}(y)$ is not commutative.

Proof. By [BB06, 2.2.6] one can choose $\gamma_{1}, \ldots, \gamma_{r} \in \Phi^{+}$such that

$$
\begin{aligned}
s_{\gamma_{r}} s_{\gamma_{r-1}} \cdots s_{\gamma_{1}} w & =y, \\
l\left(s_{\gamma_{i}} s_{\gamma_{i-1}} \cdots s_{\gamma_{1}} w\right) & =l(w)-i
\end{aligned}
$$

for all $i=1, \ldots, r$. As $y \notin W^{w}$ there exist $a, b \in\{1, \ldots, r\}, a \neq b$, such that $\left(\gamma_{a}, \gamma_{b}\right) \neq 0$. Now we may apply Corollary A.5 and assume that $\left(\gamma_{1}, \gamma_{2}\right) \neq 0$. Hence, by Proposition 3.13, the algebra $R_{0}^{w} / Q\left(s_{\gamma_{2}} s_{\gamma_{1}} w, w\right)_{w}$ is not commutative. By (3.27) we have $y \leq s_{\gamma_{2}} s_{\gamma_{1}} w$ and hence $Q(y, w)_{w} \subseteq Q\left(s_{\gamma_{2}} s_{\gamma_{1}} w, w\right)_{w}$. Thus $R_{0}^{w} / Q(y, w)_{w}$ is also not commutative which by (3.23) and (3.24) proves the proposition.

Recall that for any $\Theta \in T^{w}$ we have defined $P_{\Theta}$ to be the ideal generated by $\left\{E_{\beta} \mid \beta \in \Phi_{w}^{+} \backslash \Theta\right\}$. Then $P_{\Theta} \in H-\operatorname{spec}^{\text {poly }}\left(U^{+}[w]\right)$ by Proposition 3.9.

Corollary 3.16. (1) The map $W^{w} \rightarrow H-\operatorname{spec}^{p o l y}\left(U^{+}[w]\right), y \mapsto P^{+}(y)$, is an order preserving bijection.

(2) One has $P_{\Theta}=P^{+}\left(w_{\Theta}\right)$ for all $\Theta \in T^{w}$.

(3) If $y \in W^{w}$ then $U^{+}[w] / P^{+}(y)$ is a commutative polynomial ring in $l(w)-l(y)$ variables. These variables can be chosen to be the $E_{\beta}$ with $\beta \in\left(\varkappa^{w}\right)^{-1}(y)$.

Proof. The map in (1) is order preserving and injective because the map $W \leq w \rightarrow$ $H-\operatorname{spec}\left(U^{+}[w]\right), y \mapsto P^{+}(y)$, is an order preserving bijection. It follows from Proposition 3.9 that

$$
\left|T^{w}\right| \leq\left|H-\operatorname{spec}^{\text {poly }}\left(U^{+}[w]\right)\right| .
$$

On the other hand Proposition 3.15, together with the bijection between $W \leq w$ and the set of $H$-prime ideals in $U^{+}[w]$, implies that

$$
\left|H-\operatorname{spec}^{\text {poly }}\left(U^{+}[w]\right)\right| \leq\left|W^{w}\right| .
$$

By Lemma 3.14 one obtains $\left|W^{w}\right|=\left|H-\operatorname{spec}^{\text {poly }}\left(U^{+}[w]\right)\right|$ which shows that the map in (1) is bijective. 
By Proposition 3.9 the map $T^{w} \rightarrow H-$ spec $^{\text {poly }}\left(U^{+}[w]\right), \Theta \mapsto P_{\Theta}$ is injective. By Lemma 3.14 and (1) we conclude that

$$
\left\{P_{\Theta} \mid \Theta \in T^{w}\right\}=\left\{P^{+}(y) \mid y \in W^{w}\right\} .
$$

To prove (2) it hence suffices to show that $E_{\beta} \notin P^{+}\left(w_{\Theta}\right)$ for all $\Theta \in T^{w}$ and $\beta \in \Theta$. Let $\Theta \in T^{w}$ and $\beta \in \Theta$. Then $w_{\Theta} \leq s_{\beta} w$ by Lemma 3.8 and hence $P^{+}\left(w_{\Theta}\right) \subseteq P^{+}\left(s_{\beta} w\right)$. Thus we only need to show that $E_{\beta} \notin P^{+}\left(s_{\beta} w\right)$. To this end recall that by definition $P^{+}\left(s_{\beta} w\right)=\omega\left(\phi_{w}\left(Q\left(s_{\beta} w, w\right)_{w}\right)\right)$. Hence $\omega\left(\phi_{w}\left(c_{w}^{-\lambda} c_{s_{\beta} w}^{\lambda}\right)\right) \notin$ $P^{+}\left(s_{\beta} w\right)$ by Corollary [3.12. By definition of $\phi_{w}$ (cf. [Yak09, Theorem 3.7]) the element $\omega\left(\phi_{w}\left(c_{w}^{-\lambda} c_{s_{\beta} w}^{\lambda}\right)\right)$ belongs to the weight space of weight $m \beta$ of $U^{+}[w]$ for some $m \in \mathbb{N}_{0}$. We may assume $m \neq 0$ by choosing $\lambda \in \Lambda^{+}$regular. Hence $U^{+}[w] / P^{+}\left(s_{\beta} w\right)$ contains an element of weight $m \beta$ for some positive integer $m$.

On the other hand it follows from (1) and Equation (3.30) that $P^{+}(w)=P_{\emptyset}$ and that $P^{+}\left(s_{\beta} w\right)=P_{\{\gamma\}}$ for some $\gamma \in \Phi_{w}^{+}$with $\{\gamma\} \in T^{w}$. Proposition 3.9 hence implies that $U^{+}[w] / P^{+}\left(s_{\beta} w\right)$ is a polynomial ring in $E_{\gamma}$. Therefore $\beta=\gamma$ and $E_{\beta} \notin P^{+}\left(s_{\beta} w\right)$ which completes the proof of (2). Property (3) follows immediately from (2) and Proposition 3.9 .

With the above corollary we have all the information necessary to give an explicit description of the characters of $U^{+}[w]$. For any two sets $A, B$ let $\operatorname{Map}(A, B)$ denote the set of maps from $A$ to $B$. As before, let $k^{\times}=k \backslash\{0\}$. Recall also the definition of $\varkappa^{w}$ from Equation (3.25).

Theorem 3.17. There is a bijection

$$
\Psi:\left\{(y, f) \mid y \in W^{w}, f \in \operatorname{Map}\left(\left(\varkappa^{w}\right)^{-1}(y), k^{\times}\right)\right\} \rightarrow \operatorname{Char}\left(U^{+}[w]\right)
$$

uniquely determined by

$$
\Psi(y, f)\left(E_{\beta}\right)= \begin{cases}f(\beta) & \text { if } \beta \in\left(\varkappa^{w}\right)^{-1}(y), \\ 0 & \text { otherwise. }\end{cases}
$$

The inverse map is given by $\Psi^{-1}(\phi)=\left(w_{\Phi_{w}^{+}(\phi)}, f_{\phi}\right)$ for all $\phi \in \operatorname{Char}\left(U^{+}[w]\right)$, where $f_{\phi}(\beta)=\phi\left(E_{\beta}\right)$ for all $\beta \in \Phi_{w}^{+}(\phi)$.

For the classification of right coideal subalgebras in Theorem 2.15 it is necessary to determine subgroups $L$ of the root lattice which are orthogonal to supp $\phi$ for a given character $\phi$ of $U^{+}[w]$. To this end we also note the following immediate consequence of Proposition 3.3 .

Proposition 3.18. Let $\phi \in \operatorname{Char}\left(U^{+}[w]\right)$. Then $\operatorname{supp} \phi=\sum_{\beta \in \Phi_{w}^{+}(\phi)} \mathbb{N}_{0} \beta$.

\section{Appendix A. Root systems AND Weyl group COMBINATORICS}

We collect here some auxiliary results about root systems and the Bruhat order for finite Weyl groups, which are used to obtain the combinatorial classification of characters of $U^{+}[w]$ in Section 3 . First we state two lemmata on roots.

Lemma A.1. Hum72, 9.4] Let $\alpha, \beta \in \Phi$. If $(\alpha, \beta) \neq 0$ and $(\alpha, \alpha) \leq(\beta, \beta)$ then $2|(\alpha, \beta)|=(\beta, \beta)$.

Lemma A.2. Let $\gamma_{1}, \ldots, \gamma_{s}, \beta, \beta^{\prime} \in \Phi^{+}$and $a_{1}, \ldots, a_{s} \in \mathbb{N}_{0}$. Assume that $\beta+\beta^{\prime}=$ $\sum_{m=1}^{s} a_{m} \gamma_{m}$. Then there exists $n \in\{1, \ldots, s\}$ with $a_{n}\left(\gamma_{n}, \beta\right) \neq 0$ or $a_{n}\left(\gamma_{n}, \beta^{\prime}\right) \neq 0$.

Proof. This follows from $\left(\beta+\beta^{\prime}, \beta+\beta^{\prime}\right) \neq 0$. 
Now we turn to the well-known characterization of the Bruhat order in terms of positive roots and regular weights. Recall that $\lambda \in \Lambda^{+}$is called regular if $(\alpha, \lambda)>0$ for all simple roots $\alpha \in \Pi$.

Lemma A.3. Let $\lambda \in \Lambda^{+}$be a regular weight, $u \in W$, and $\beta \in \Phi^{+}$. Then the following are equivalent: (1) $s_{\beta} u<u, \quad$ (2) $u^{-1} \beta \in \Phi^{-}, \quad$ (3) $(\beta, u \lambda)<0$.

Proof. The equivalence of (1) and (2) is an immediate consequence of the strong exchange condition, cf. [BB06, 1.4.3, 4.4.6]. The equivalence of (2) and (3) follows from the $W$-invariance of the bilinear form $(\cdot, \cdot)$.

The following technical result is used in the proof of Proposition 3.15,

Lemma A.4. Let $\alpha, \beta, \gamma \in \Phi^{+}$and $w \in W$ such that

$$
l\left(s_{\alpha} s_{\beta} s_{\gamma} w\right)=l\left(s_{\beta} s_{\gamma} w\right)-1=l\left(s_{\gamma} w\right)-2=l(w)-3 .
$$

If $(\alpha, \beta) \neq 0$ or $(\alpha, \gamma) \neq 0$ then there exist $\alpha^{\prime}, \beta^{\prime}, \gamma^{\prime} \in \Phi^{+}$with $\left(\beta^{\prime}, \gamma^{\prime}\right) \neq 0$ such that $s_{\alpha} s_{\beta} s_{\gamma}=s_{\alpha^{\prime}} s_{\beta^{\prime}} s_{\gamma^{\prime}}$ and

$$
l\left(s_{\alpha^{\prime}} s_{\beta^{\prime}} s_{\gamma^{\prime}} w\right)=l\left(s_{\beta^{\prime}} s_{\gamma^{\prime}} w\right)-1=l\left(s_{\gamma^{\prime}} w\right)-2=l(w)-3 .
$$

Proof. It suffices to consider the case with $(\beta, \gamma)=0$.

If $(\alpha, \beta)=0$ then $s_{\alpha} s_{\beta}=s_{\beta} s_{\alpha}$ and we are done with $\alpha^{\prime}=\beta, \beta^{\prime}=\alpha$, and $\gamma^{\prime}=\gamma$. Exchanging $\beta$ and $\gamma$ if necessary we may hence assume that

$$
(\alpha, \beta) \neq 0 \neq(\alpha, \gamma) \text {. }
$$

Define $v=s_{\gamma} w$.

Case 1: $v^{-1} \alpha<0$. By Lemma A.3 we have $l\left(s_{\alpha} v\right)<l(v)$. It remains to show that

$$
l\left(s_{s_{\alpha} \beta} s_{\alpha} v\right)<l\left(s_{\alpha} v\right)
$$

because then $\alpha^{\prime}=s_{\alpha} \beta, \beta^{\prime}=\alpha$, and $\gamma^{\prime}=\gamma$ fulfill the conditions of the lemma. For any regular $\lambda \in \Lambda^{+}$one has $\left(s_{\alpha} \beta, s_{\alpha} v \lambda\right)=(\beta, v \lambda)<0$ by Lemma A.3 and the second equality in A.1. Again by Lemma A.3 this proves A.3.

Case 2: $v^{-1} \alpha>0$. We first claim that $(\alpha, \beta)<0$. Indeed, by the first equality in (A.1) one has for any regular $\lambda \in \Lambda^{+}$the relation

$$
0>\left(\alpha, s_{\beta} v \lambda\right)=(\alpha, v \lambda)-\frac{2(\alpha, \beta)}{(\beta, \beta)}(\beta, v \lambda) .
$$

As $s_{\beta} v<v$ one has $(\beta, v \lambda)<0$. Using $(\alpha, v \lambda)>0$, which holds by assumption, one now gets the desired $(\alpha, \beta)<0$ from (A.4).

Case 2a: $v^{-1} \alpha>0$ and $(\alpha, \alpha)=(\beta, \beta)=-2(\alpha, \beta)$. This is the case whenever $\alpha$ and $\beta$ have the same length. As in Case 1 one verifies that

$$
s_{\beta} s_{s_{\beta} \alpha} v<s_{s_{\beta} \alpha} v<v .
$$

Indeed, $\left(\alpha, s_{\beta} v \lambda\right)<0$ for any regular $\lambda \in \Lambda^{+}$by (A.1) and Lemma A.3. Hence $\left(s_{\beta} \alpha, v \lambda\right)<0$ which again by Lemma A.3 implies $s_{s_{\beta} \alpha} v<v$. To obtain the first relation in (A.5) note that $s_{\beta} \alpha=s_{\alpha} \beta=\alpha+\beta$ and hence

$$
s_{s_{\beta} \alpha} \beta=s_{\beta} s_{\alpha} s_{\beta} \beta=-\alpha .
$$

Now the first relation in (A.5) follows from Lemma A.3 and

$$
\left(\beta, s_{s_{\beta} \alpha} v \lambda\right)=(-\alpha, v \lambda)<0 .
$$


Case 2b: $v^{-1} \alpha>0$ and $(\alpha, \alpha) \neq(\beta, \beta)$. Recall that $(\alpha, \beta)<0$. Since $\beta$ and $\gamma$ may be exchanged, we can assume that $(\beta, \beta) \neq(\alpha, \alpha) \neq(\gamma, \gamma)$ and $(\alpha, \gamma)<0$, $(\alpha, \beta)<0$, and $(\beta, \gamma)=0$. This, however, is impossible in a finite root system. Indeed, if $(\beta, \beta)=2$ and $(\alpha, \alpha)=2 d$ with $d=2$ or $d=3$, then $z:=\alpha+\beta+\gamma$ is a root. Moreover, this root satisfies $(z, \alpha)=0$ and $(z, \beta)=(z, \gamma) \leq 0$ by Lemma A.1. This yields the contradiction $(z, z) \leq 0$. Similarly, one obtains a contradiction if $\beta$ is long and $\alpha$ is short by considering $2 \alpha+\beta+\gamma$.

Lemma 3.8 and repeated application of Lemma A.4 imply the following Corollary.

Corollary A.5. Let $w \in W$ and assume that $\beta_{1}, \ldots, \beta_{m} \in \Phi^{+}$satisfy

$$
l\left(s_{\beta_{i}} s_{\beta_{i-1}} \cdots s_{\beta_{1}} w\right)=l(w)-i
$$

for all $i=1, \ldots, m$. Assume, moreover, that there exist $i, j \in\{1, \ldots, m\}, i \neq j$, such that $\left(\beta_{i}, \beta_{j}\right) \neq 0$. Then there exist $\gamma_{1}, \ldots, \gamma_{m} \in \Phi^{+}$such that

$$
\begin{aligned}
l\left(s_{\gamma_{i}} s_{\gamma_{i-1}} \cdots s_{\gamma_{1}} w\right) & =l(w)-i \quad \text { for all } i \in\{1, \ldots, m\}, \\
s_{\gamma_{m}} s_{\gamma_{m-1}} \cdots s_{\gamma_{1}} w & =s_{\beta_{m}} s_{\beta_{m-1}} \cdots s_{\beta_{1}} w,
\end{aligned}
$$

and $\left(\gamma_{1}, \gamma_{2}\right) \neq 0$.

\section{REFERENCES}

[BB06] A. Björner and F. Brenti, Combinatorics of Coxeter groups, Springer-Verlag, New York, 2006.

[BG02] K. A. Brown and K. G. Goodearl, Lectures on algebraic quantum groups, Birkäuser Verlag, Basel, 2002.

[CKP95] C. De Concini, V.G. Kac, and C. Procesi, Some quantum analogues of solvable Lie groups, Geometry and analysis (Bombay 1992) (Bombay), Tata Inst. Fund. Res., 1995, pp. $41-65$.

[Dij96] M.S. Dijkhuizen, Some remarks on the construction of quantum symmetric spaces, Acta Appl. Math. 44 (1996), no. 1-2, 59-80.

[Gor00] M. Gorelik, The prime and primitive spectra of a quantum Bruhat cell translate, J. Algebra 227 (2000), 211-253.

[HS09] I. Heckenberger and H.-J. Schneider, Right coideal subalgebras of Nichols algebras and the Duflo order on the Weyl groupoid, Preprint, arXiv:0909.0293 (2009), 43 pp.

[Hum72] J. E. Humphreys, Introduction to Lie algebras and representation theory, SpringerVerlag, New York, 1972.

[Jan96] J.C. Jantzen, Lectures on quantum groups, Grad. Stud. Math., vol. 6, Amer. Math. Soc, Providence, RI, 1996.

[Jos95] A. Joseph, Quantum groups and their primitive ideals, Ergebnisse der Mathematik und ihrer Grenzgebiete, Springer-Verlag, Berlin, 1995.

[Kha09] V. Kharchenko, Right coideal subalgebras in $U_{q}^{+}\left(\mathfrak{s o}_{2 n+1}\right)$, Preprint, arXiv:0908.4235 (2009), 53 pp.

[Koo93] T. Koornwinder, Askey-Wilson polynomials as zonal spherical functions on the SU(2) quantum group, SIAM J. Math. Anal. 24 (1993), no. 3, 795-813.

[KS08] V. Kharchenko and A.V.L. Sagahon, Right coideal subalgebras in $U_{q}^{+}\left(\mathfrak{s l}_{n+1}\right)$, J. Algebra 319 (2008), 2571-2625.

[KS09] S. Kolb and J. Stokman, Reflection equation algebras, coideal subalgebras, and their centres, Selecta Math. (N.S.), online first (2009), DOI: 10.1007/s00029-009-0007-1.

[Let02] G. Letzter, Coideal subalgebras and quantum symmetric pairs, New directions in Hopf algebras (Cambridge), MSRI publications, vol. 43, Cambridge Univ. Press, 2002, pp. 117166.

[Let03] - Quantum symmetric pairs and their zonal spherical functions, Transformation Groups 8 (2003), 261-292.

[LS91] S. Levendorskiî and Y. Soibelman, Algebras of functions on compact quantum groups, Schubert cells and quantum tori, Commun. Math. Phys. 139 (1991), no. 1, 141-170. 
[Mon93] S. Montgomery, Hopf algebras and their actions on rings, AMS, Providence, Rhode Island, 1993.

[NDS97] M. Noumi, M.S. Dijkhuizen, and T. Sugitani, Multivariable Askey-Wilson polynomials and quantum complex Grassmannians, AMS Fields Inst. Commun. 14 (1997), 167-177.

[Nou96] M. Noumi, Macdonald's symmetric polynomials as zonal spherical functions on some quantum homogeneous spaces, Adv. Math. 123 (1996), 16-77.

[Pog09] B. Pogorelsky, Right coideal subalgebras of the quantum Borel subalgebra of type $G_{2}$, J. Algebra 322 (2009), 2335-2354.

[Yak09] M. Yakimov, Invariant prime ideals in quantizations of nilpotent Lie algebras, Preprint arXiv:0905.0852 (2009), 25 pp.

István Heckenberger, Fachbereich Mathematik, Philipps-Universität Marburg, HansMeerwein-Strasse, 35032 Marburg, Germany

E-mail address: heckenberger@mathematik.uni-marburg.de

Stefan Kolb, Korteweg-de Vries Institute for Mathematics, University of Amsterdam, Science Park 904, 1098 XH Amsterdam, The Netherlands

E-mail address: s.kolb@uva.nl 